ESJ Social Sciences

\title{
Evaluation des Effets des Accords Euro-Mediterraneens sur les Echanges Commerciaux Marocains: Enseignements d'un Modele de Gravite
}

\author{
Zineb Mikdam, (Docteur en sciences Economiques et Gestion)
}

Ecole Nationale du Commerce et de Gestion de Meknès, Maroc

Doi:10.19044/esj.2021.v17n38p102

Submitted: 05 April 2021

Accepted: 30 October 2021

Published: 30 November 2021
Copyright 2021 Author(s)

Under Creative Commons BY-NC-ND

4.0 OPEN ACCESS

Cite As:

Mikdam Z. (2021). Evaluation des Effets des Accords Euro-Mediterraneens sur les Echanges Commerciaux Marocains: Enseignements d'un Modele de Gravite. European Scientific Journal, ESJ, 17 (38), 102. https://doi.org/10.19044/esj.2021.v17n38p102

\section{Résumé}

Cet article s'intéresse à l'étude de l'impact du processus d'intégration du Maroc dans l'espace euro-méditerranéen en particulier en termes d'échanges commerciaux, en examinant les effets de la politique libreéchangiste adoptée par le Maroc dans l'espace euro-méditerranéen et son intérêt dans la promotion du potentiel commercial du pays. A travers une modélisation économétrique basée sur l'approche gravitationnelle qui semble être d'une grande utilité, l'étude a permis de distinguer entre les effets des accords de libre-échange conclus par le Maroc avec les pays arabes méditerranéens et la Turquie d'une part, et avec l'UE d'autre part. De même, ce modèle a permis d'apprécier l'intérêt de la variable gravitationnelle à savoir, la distance et ses effets explicatifs du commerce marocain intrarégional. Il est à noter d'après cette étude économétrique que les accords de libre-échange ont certainement contribué à la croissance des échanges du Maroc avec ses partenaires méditerranéens, alors que d'autres facteurs, à savoir la distance, le taux de change et l'existence d'une langue commune influencent positivement mais d'une manière négligeable les échanges commerciaux du Maroc dans l'espace euro-méditerranéen. Ainsi, et afin de consolider les effets positifs de ces accords de libre-échange, le pays doit adopter stratégies internes de promotion des secteurs économiques à fort potentiel d'exportation dans la perspective de stimuler ses exportations vers ses partenaires euro-méditerranéens. 
Mots clés : Intégration, Partenariat, Libre-échange, Modèle de gravité

\title{
Evaluation of the Effects of Euro-Mediterranean Agreements on Moroccan Trade: Lessons From a Gravity Model
}

\author{
Zineb Mikdam, (Docteur en sciences Economiques et Gestion) \\ Ecole Nationale du Commerce et de Gestion de Meknès, Maroc
}

\begin{abstract}
This article focuses on studying the impact of Morocco's integration process in the Euro-Mediterranean area, particularly in terms of trade, by examining the effects of the free trade policy adopted by Morocco in the EuroMediterranean area and its implication in promoting the country's commercial potential. Through an econometric model based on the gravitational approach which seems to be of great use, the study made it possible to distinguish between the effects of the free trade agreements concluded by Morocco with the Mediterranean Arab countries and Turkey on the one hand and with the EU on the other hand. Moreover, this model helped to appreciate the interest of the gravitational variable namely the distance and its explanatory effects of intra-regional Moroccan trade. We note from this econometric study that free trade agreements have certainly contributed to the growth of Morocco's trade with its Mediterranean partners, while other factors, which are the distance, the exchange rate and the existence of a common language, influence in a negligible positive way the commercial exchanges of Morocco in the EuroMediterranean area. In order to consolidate the positive effects of these free trade agreements, Morocco must adopt internal strategies that aim to promote economic sectors with high export potential which can lead to stimulating its exports toward its Euro-Mediterranean partners.
\end{abstract}

Keywords: Integration, Partnership, Free trade, Gravity Approach

\section{Introduction}

La mouvance de la mondialisation des relations économiques internationales au cours de ces dernières années est devenue une contrainte. Ainsi, le Maroc s'est trouvé dans la nécessité de se tourner vers les marchés étrangers, en faisant de l'ouverture un choix stratégique inéluctable. Donc, le pays a pris le parti de s'intégrer dans l'économie mondiale dans son nouveau contexte. 
De ce fait, cette ouverture se trouve renforcée par l'adhésion du Maroc à l'Organisation Mondiale du Commerce et les accords de libre-échange conclus avec l'Union Européenne, et certains pays arabes de l'espace euroméditerranéen ainsi qu'avec la Turquie. Cette ouverture constitue une solution concevable à une croissance soutenue et durable, et même un défi incontestable pour le Maroc, qui accélère sa tendance à la mondialisation, la régionalisation et l'intégration dans son espace euro-méditerranéen, en renforçant ses relations avec les pays européens et les pays arabes de cet espace.

Dans cette perspective, nous ne recherchons pas seulement à confronter les théories de l'intégration économique au réalisme de la sousrégion euro-méditerranéenne, dans laquelle le Maroc joue un rôle majeur, mais surtout à montrer les effets de la conclusion des accords de libre-échange, représentés dans notre étude par les flux des échanges commerciaux marocains, en jouant sur cet instrument qui est la régionalisation.

Certainement, le Maroc opère dans un espace large dans le cadre d'un partenariat, qui a rassemblé les deux (2) rives de la méditerranée. Cependant, le Maroc a opté pour des accords bilatéraux, en favorisant le libre-échange comme forme d'intégration qui le relie à ces pays. Il nous semble donc évident d'évaluer les relations du Maroc avec ces pays, étant donné qu'il existe une forme d'intégration qui les rassemble.

Afin d'évaluer l'impact commercial de différents accords de libreéchange, les études économiques se fient depuis les années 1960 au modèle de gravité, tel est le cas de Tinbergen (1962) et Poyhonen (1963). Ce modèle est utilisé à la base pour expliquer les principaux facteurs, qui influencent le volume et la direction du commerce international d'un pays. En fait, le modèle de gravité a été employé en économie pour expliquer les différentes extensions du commerce international, et pour obtenir des estimations plus fiables sur les flux commerciaux internationaux.

Notre étude prendra en compte les développements récents dans la technique d'estimation de gravité, pour étudier les déterminants des échanges commerciaux du Maroc dans un cadre de données de panel.

\section{Modélisation économétrique par gravité en données de panel}

Le modèle de gravité que nous avons construit se base sur des données de panel sur une période de onze (11) ans ${ }^{1,}$ et d'un échantillon composé des

\footnotetext{
${ }^{1}$ Nous avons pris en considération les statistiques de l'année 2000 jusqu'à la fin de l'année 2011, puisque le plus ancien accord de libre-échange adopté par le Maroc dans l'espace euroméditerranéen est celui conclu avec l'UE. En fait, nous avons opté pour les statistiques jusqu'à l'année 2011 car les données statistiques sont disponibles jusqu'à la fin de 2011 d'une part, et puisque le début de l'année 2012 correspond à l'arrivée à terme du processus du démantèlement douanier notamment avec l'UE d'autre part. Donc, nous nous sommes basés
} 
pays de $1^{\prime} \mathrm{UE}^{2}$, et certains pays arabes méditerranéens (Jordanie, Tunisie, Egypte), ainsi que la Turquie. Donc, notre modèle est composé des 31 pays de l'espace euro-méditerranéen, avec lesquels le Maroc a conclu des accords de libre-échange.

En fait, les principes des modèles de gravité nous semblent être plus adéquats à l'analyse que nous voulons mener.

D'ailleurs, plusieurs moyens peuvent être adoptés pour évaluer le degré du processus d'intégration d'un pays dans un espace régional. Dans notre étude, nous avons choisi les flux des échanges commerciaux comme instrument de mesure, car il nous semble qu'ils sont plus illustratifs du processus d'intégration.

\section{I.1. Fondements théoriques du modèle de gravité}

Parmi les techniques d'évaluation des échanges régionaux, on considère que le modèle de gravité est la méthode la plus simple qui donne souvent de bons résultats pour prédire le volume du commerce bilatéral.

En fait, les modèles de gravité permettent d'expliquer empiriquement une relation commerciale entre deux (2) pays, dans la mesure où nous pouvons ajouter ou éliminer des variables. Ce modèle est une appellation générique de la famille de modèles quantitatifs développés en 1940, et qui a connu un large succès empirique depuis les années 60 . Et pourtant ce modèle a souffert sur le plan économique, d'une absence de fondements théoriques.

Néanmoins, il nous semble que le modèle gravitationnel est l'un des modèles le plus vigoureux, d'un point de vue économétrique, pour prédire et expliquer les courants d'échange bilatéraux, puisqu'il permet de mettre en évidence les échanges entre deux (2) pays en fonction de leurs PIB respectifs - comme approximation de la puissance économique des pays- et de la distance qui les séparent -comme estimation des coûts de transport. Conformément à ce modèle, l'intensité des échanges entre deux (2) pays est proportionnelle au produit de leurs PIB, et inversement proportionnelle à la distance qui les sépare.

De ce fait, la présence de la composante géographique implique que l'espace compte dans les phénomènes économiques au travers de la distance séparant les pays. Les pays ne sont plus considérés comme des points situés

sur cet accord pour rassembler les données statistiques de notre étude puisque même les accords euro-méditerranéens conclu par le Maroc ont eu lieu dans cette période.

${ }^{2}$ Nous avons pris en considération les pays de l'UE des 27 avant l'adhésion de la Croatie en 2013, car les données relatives à l'année 2012 et 2013 n'étaient pas encore disponibles dans les organismes qui nous ont fournis les statistiques. Rappelons à cet égard que l'année 2012, est marquée par la fin du démantèlement douanier progressif, et la zone de libre-échange Maroc-UE pour les produits industriels est devenue effective, donc les organismes ne possèdent pas encore les statistiques exactes avec la fin du démantèlement. 
dans l'espace sans distance mais comme des entités géographiques, dont la localisation relative compte et nécessite des coûts de transaction lors des échanges. Alors, l'idée d'appliquer un modèle de gravité repose également sur l'optique de prendre en considération ces coûts et ces positions géographiques qui peuvent être stratégiques et vitales pour une économie dans ses rapports avec l'extérieur.

Toutefois, le modèle de gravité a permis à certains économistes d'expliquer la structure de différents exemples de commerce international. Citons, parmi d'autres, son application à l'interprétation des échanges commerciaux entre l'UE et le MERCOSUR, à la prédiction des flux commerciaux entre les pays baltes et leurs partenaires, et à l'exploration du commerce international des pays candidats à l'intégration dans l'UE'.

De même, nous avons utilisé des données de panel dans notre modèle, afin d'enrichir l'information que nous allons obtenir tant en quantité qu'en variabilité. D'un point de vue économétrique, cette richesse conduit à une grande précision des estimations. Elle permet aussi de considérer que les résultats obtenus sont « asymptotiques ».

En effet, nous avons opté pour des données en panel car elles permettent le contrôle des facteurs qui varient entre les individus, mais ne varient pas au cours du temps, et qui pourraient causer un biais d'omission si l'on en tenait pas compte, et qui sont inobservables ou non disponibles et ne peuvent être inclus dans la régression ${ }^{4}$.

De même, notre étude diffère des précédents travaux, puisque nous allons utilisé des modèles adaptés au cas de la région méditerranéenne, qui repose sur l'élaboration d'un modèle de gravité qui tient compte des altérités du bassin méditerranéen et les spécificités nationales pour expliquer l'évolution du commerce extérieur marocain intra-zone, en tenant compte des caractéristiques du modèle gravitationnel en tant qu'approche économétrique post-intégration qui implique des facteurs autres qu'économiques comme éléments d'explication des échanges intra-régionaux.

\section{I.2. Modèle gravitationnel proposé}

Deux (2) études récentes, de McCallum en 1995 et Haris et Mátyás en 1998 sont particulièrement liées à notre étude.

En fait, McCallum a étudié les flux commerciaux transfrontaliers aux Etats-Unis et du Canada en utilisant les données de 1988 pour toutes les dix provinces du Canada et des trente Etats des Etats-Unis.

\footnotetext{
${ }^{3}$ LEBLOND P., ANDREA STRACHINESCU-OLTEANU M. (2009), Le libre-échange avec l'Europe : quel est l'intérêt pour le Canada, In Canadian Foreign Policy Vol $15 \mathrm{~N}^{\circ} 1$.

${ }^{4}$ JUILLARD M (2007), Cours d'économétrie II : Données de panel, organisé par L'UMRParis-Jourdan Sciences Economiques le 27 Mars 2007.
} 
Il a constaté que suite au partage des frontières communes entre le Canada et les Etats Unis, le commerce intérieur entre les provinces canadiennes est vingt-deux (22) fois plus grand que le commerce transfrontalier entre les provinces canadiennes et les États américains. Il a également montré que les coûts de transport jouent un rôle important dans l'explication de la structure des échanges.

Harris et Mátyás (1998), ont fait quant à eux une tentative formelle pour améliorer le modèle de gravité de base. En fait, ils ont montré que le modèle de base ne tient pas compte des variables explicatives importants tels que les taux de change étrangers, et les réserves en devises. Ils ont également constaté que les flux d'exportation actuels étaient fortement liés à ceux de l'année précédente.

Nous essayerons de déterminer les effets de différents facteurs pris en considération, dans le cadre du processus d'intégration entamé par le Maroc représentés par les flux commerciaux avec ses partenaires méditerranéens, en tenant compte de toutes les variables ci-dessus. Nous nous appuierons sur l'équation de gravité de McCallum (1995) avec quelques modifications telles que proposées par Harris et Mátyás (1998) pour dériver une spécification plus appropriée. Plus précisément, nous estimons notre modèle à travers un modèle de panel statique et un modèle de panel dynamique.

Dans notre démarche de sélection des variables, nous allons opter pour des variables dites traditionnelles de l'équation de gravité pour ensuite les compléter par des variables supplémentaires prises de manière bilatérale.

L'enjeu n'est pas seulement de modéliser les échanges bilatéraux, en terme commercial dans le cadre des relations du Maroc avec les pays méditerranéens. Nous allons en effet faire en sorte que le modèle soit conforme à la situation commerciale de la région. Ainsi, aux effets habituels des exportations, importations de distance et de frontière, nous ajouterons d'autres effets comme la variation du taux de change.

En effet, la variable dépendante $\mathrm{Y}_{\mathrm{ij}}$ que nous allons prendre en considération est présentée par les échanges commerciaux (exportations et importations) du Maroc vers ses partenaires méditerranéens. D'ailleurs, Frankel en 1997, avait suggéré l'observation des flux des exportations et des importations séparés dans un modèle de gravité, étant donné que l'appréciation de la monnaie d'un pays engendre une diminution de la demande de ses produits, et par conséquent une baisse de ses exportations, tandis qu'elle stimule l'augmentation des importations.

Dans le même sens, la somme des deux (2) flux commerciaux ne permettra pas de mettre en évidence l'existence de la balance commerciale et l'influence de certains facteurs macroéconomiques, précisément l'influence du taux de change sur le volume des flux commerciaux (Krugman et Obstfeld, 2003), qui a un impact différent sur les flux commerciaux, puisque 
l'appréciation de la monnaie entraine une augmentation des prix, ainsi qu'une diminution de la demande, et par conséquent, une diminution de ses exportations, et une croissance des importations. Donc l'utilisation des échanges globaux en tant que variable dépendante ne permettra pas d'obtenir des résultats pertinents, et une pratique courante dans les études de gravitation.

Dans ce cadre, nous nous sommes référés à la base des données de l'Office des changes du Maroc pour la période 1997 à 2011 exprimées en dirhams marocain, afin d'obtenir les valeurs des importations et des exportations. Nous avons pris en considération que la balance des paiements doit être établie selon la méthodologie du manuel édicté par le Fond Monétaire International, permettant ainsi à l'Office des changes d'adapter le dispositif d'établissement des statistiques des échanges extérieurs aux nouvelles normes internationales en vigueur, où les importations marocaines doivent comprendre également les admissions temporaires sans paiement. Notre objectif est d'avoir une homogénéité de l'unité de mesure utilisée et la validité des résultats.

Rappelons que nous avons utilisé le dirham marocain, afin d'avoir une uniformité de l'unité de mesure puisque la monnaie unique européenne ${ }^{5}$ «l'Euro » adoptée par les pays de l'Union est apparue officiellement dans les transactions financières européenne en 1999 dans les 27 pays de l'UE, devenue l'UE des 28 après l'adhésion de la Croatie en 2013.

En fait, nous allons développer notre modèle en nous basant sur des données en panel, en tenant compte de l'impact du taux de change sur le volume du commerce, ainsi que l'impact de l'adoption d'une relation commerciale préférentielle (création d'une zone de libre-échange) à travers une variable muette ${ }^{6}$. De même, notre équation de gravité comprend la distance géographique entre les partenaires commerciaux, qui représentent des coûts de transaction, comme une variable explicative.

Notre modèle comprend également la variable fondamentale de gravitation, représentée par le PIB par habitant «PIB en PPA » des partenaires commerciaux, qui permettra de représenter le poids du pays dans le commerce international. De même, nous avons retenu le PIB en parité du pouvoir d'achat (PPA) dans notre étude, car l'influence de certains facteurs, notamment le taux d'intérêt et les flux des capitaux, demeure assez importante sur les prix relatifs

\footnotetext{
${ }^{5}$ Il est à noter que la Turquie a adopté une nouvelle monnaie en 2005 (la nouvelle Lire), suite aux différentes crises qu'a connu l'ancienne monnaie notamment en 1999 et 2001, avec des conséquences néfastes sur le secteur bancaire, l'endettement du pays et sa croissance économique. Ce changement de la monnaie turc en 2005 a été pris en considération dans notre étude.

${ }^{6}$ C'est une variable binaire ou dummy qui ne peut prendre que deux (2) valeurs, le plus souvent associées à $\{0,1\}$ ou $\{$ absence, présence $\}$.
} 
des biens et investis plus que le taux de change, qui ne reflète que partiellement leurs variations et à courte période.

Ainsi, à l'instar de toutes les données que nous utilisons, les valeurs du PIB en (PPA) sont exprimées en dirhams marocain, en effectuant la conversion de l'Euro sur la base du taux de change. Ces données ont été obtenues de la base des données de «Eurostat: Statistical Office of the European Commission» de la commission européenne, et l'office des changes du Maroc, en comparant avec les données de la Banque mondiale exprimées en dollars américain.

Une autre variable que nous allons prendre en considération, le taux de change effectif entre la monnaie du pays i (le Dirham) et le pays j (l'Euro), en tant que variable de contrôle. En fait, l'accord de libre-échange Maroc-UE est entré en vigueur en 2000, et l'UE a connu un élargissement entre 2000 à 2012, de l'Union des quinze (15), des vingt-cinq (25), jusqu'à l'Union des vingt-sept (27), et en 2013, l'UE des vingt-huit (28) avec l'adhésion de la Croatie. Cependant, notre étude porte sur un délai de onze ans, entre 2000 et 2011, vu la disponibilité des données dans cette période et la fin du démantèlement douanier au début de l'année 2012.

\section{I.3. Equations proposées}

Les deux (2) équations de base des échanges commerciaux (exportations et importations), que nous allons construire pour les données de panels de notre thèse se présentent sous la forme générale suivante:

$$
y_{j, t}=\alpha_{j}+\mu_{j} x_{j, t}+\varepsilon_{j, t}
$$

avec : $y_{j, t}$ : les variables à expliquer ; $\alpha_{j}$ : une constante ; $\mu_{j}$ : les paramètres à estimer $; x_{j, t}:$ les variables explicatives ; $\varepsilon_{j, t}:$ les erreurs

La signification de l'équation générale par rapport au modèle des exportations est la suivante :

- La variable à expliquer, $y_{j, t}$, devient $\mathrm{X}_{\mathrm{jt}}$ qui représente les exportations du Maroc vers le pays partenaire méditerranéen $\mathrm{j}$, exprimées en Dirham ;

- Le vecteur des variables explicatives $x_{j, t}$ est composé des 8 variables suivantes :

- PIB.Mat : le revenu du Maroc mesuré par le PIB en parité du pouvoir d'achat par habitant ;

- PIB $_{\mathrm{jt}}$ : le revenu du pays j mesuré par le PIB en parité du pouvoir d'achat par habitant ;

- Distance $\mathrm{j}_{\mathrm{j}}$ : la distance entre le Maroc et le pays j en kilomètres ;

- $\mathrm{TCH}_{\mathrm{jt}}$ : le taux de change réel moyen entre le Dirham marocain et la monnaie du pays $\mathrm{j}$ enregistré dans la période $\mathrm{t}$; 
- $\mathrm{ALE}_{\mathrm{t}}$ : une variable muette qui prend la valeur 1 lorsqu'il y a un accord de libre-échange entre le Maroc et le pays $\mathrm{j}$, alors qu'elle prend la valeur 0 dans le cas d'absence de cet accord ;

- $\mathrm{DMPE}_{\mathrm{jt}}$ : le droit moyen préférentiel aux exportations ;

- Lang $_{\mathrm{j}}$ : une variable muette qui prend la valeur 1 si le Maroc et le partenaire j partagent une langue commune, et 0 sinon ;

- Colon $\mathrm{j}_{\mathrm{j}}$ une variable muette qui prend la valeur 1 si le Maroc et le partenaire j ont eu ou avaient un lien de colonisation, et 0 sinon ;

La signification de l'équation générale pour le modèle des importations se présente, quant à elle, comme suit :

$\mathrm{La}$ variable à expliquer, $y_{j, t}$, devient $\mathrm{M}_{\mathrm{jt}}$ qui représente les importations du Maroc vers le pays j, exprimées en Dirham ;

Le vecteur des variables explicatives $x_{j, t}$ est composé des mêmes variables du explicatives du modèle des exportations, à l'exception du DMPE qui sera remplacé par le $\mathrm{DMPM}_{\mathrm{jt}}$ : droit moyen préférentiel aux importations.

Le test de notre modèle reposera sur un échantillon de $27^{7}$ pays de l'UE. Il tient compte de différentes étapes d'élargissement de l'UE. Nous considérons aussi les pays méditerranéens avec lesquels le Maroc a conclu des accords de libre-échange (Turquie, les pays de l'accord d'Agadir). Il s'agit alors d'un panel composé de 31 pays au total. Alors que la variable dépendante est représentée par une période de 11 ans, allant de $2000^{8}$ jusqu'à 2011 , en tenant compte des retards en tant que variables explicatives.

Notons dans ce cadre que, les accords de libre-échange conclus par le Maroc avec les pays Sud méditerranéens ont commencé en 1999, à travers des accords bilatéraux, respectivement avec la Tunisie et l'Egypte, suivi par l'accord quadripartite (accord d'Agadir) en 2004, ainsi que l'accord de libreéchange avec la Turquie en 2006. Par conséquent, la variable dépendante de notre modèle s'étalera sur la période entre l'année 2000 et 2011.

En fait, le choix de cette période de 11 ans, nous permettra d'obtenir les données de la période de progression du processus de démantèlement douanier, en utilisant les variables «DMPE » et «DMPM $»^{9}$, qui représentent

\footnotetext{
${ }^{7}$ Les pays de l'UE avant l'adhésion de la Croatie en 2013, en tant que $28{ }^{\text {ème }}$ membre, puisque nous avons opté pour une période entre 2000 et 2011. Les données de 2012 et 2013 n'étaient pas encore disponibles.

${ }^{8}$ Avec la signature de l'accord entre le Maroc et l'UE, puisque c'est l'accord le plus ancien des accords conclus par le Maroc dans l'espace euro-méditerranéen.

${ }^{9}$ Site officiel de la Délégation de l'UE au Maroc; Démantèlement douanier progressif : mesure en vigueur le 1er 2012 : www.eeas.europa.eu/delegations/morocco/eu_morocco/trade.../index_fr.htm; Consulté le $10 / 03 / 2012$.
} 
la progression de ce démantèlement après l'adoption des accords de libreéchange.

\section{Estimation des équations et l'interprétation des résultats}

Nous allons essayer d'évaluer l'intensité commerciale entre le Maroc et ses partenaires méditerranéens, afin d'apprécier l'intérêt du processus d'intégration entamé par le pays dans le cadre des accords de libre-échange conclus avec les pays de l'espace euro-méditerranéen depuis son adoption de la politique d'ouverture au début des années 80 .

Tout d'abord, nous commencerons notre test par un modèle statique sur des données en panel, en testant les effets fixes et les effets aléatoires dans nos équations. Dans une seconde étape, nous allons opter pour un modèle dynamique en intégrant des « Lags » (retards) dans nos équations.

Les résultats que nous obtiendrons de différents tests, cherchent à investiguer la relation, qui existe entre le volume et la direction des flux commerciaux du Maroc dans l'espace euro-méditerranéen, qui sont présentés dans des tableaux récapitulatifs dans ce qui suit.

\section{II.1 Modèles et méthodologie d'estimation}

Nous allons procéder à l'estimation de nos équations gravitationnelles à travers deux (2) modèles économétriques à savoir, le modèle statique et le modèle dynamique que nous allons expliquer dans ce qui suit.

Or, il convient, avant toute estimation, de vérifier la spécification homogène ou hétérogène du processus générateur des données. Autrement dit, il est primordial de procéder aux tests de spécification pour savoir, si le processus générateur des données est unique pour tous les pays, ou s'il apparaît totalement hétérogène, auquel cas l'utilisation des techniques de panel ne peut se justifier ${ }^{10}$.

Cette première étape va nous permettre, en fait, de vérifier si le modèle théorique étudié est identique pour l'ensemble des pays du bassin méditerranéen de l'échantillon, ou au contraire il existe des spécificités propres à chaque pays. Cela revient, sur le plan économétrique, à tester l'égalité des coefficients du modèle étudié dans la dimension individuelle ${ }^{11}$.

\section{II.2. Tests d'homogénéité}

Les tests d'homogénéité nous permettront de spécifier notre modèle par rapport à deux (2) principales situations :

${ }^{10}$ HURLIN C., L'économétrie des données de panel : Modèles linéaires simples, Ecole Doctorale Edocif, Séminaire méthodologique, document disponible sur le site web: www.univ-orleans.fr/deg/masters/ESA/CH/CoursPanel_Chap1.pdf, consulté le 24/06/2014.

${ }^{11}$ Ibidem. 
- Si les individus ont un comportement identique, il n'y a pas lieu de tenir compte de spécificités individuelles. Dans ce cas, nous allons privilégier une estimation par la méthode des MCO sur des données empilées.

- Or, s'il se trouve que nous avons une hétérogénéité des comportements, l'estimation par la méthode des MCO ne sera plus appropriée tant qu'elle ignore les spécificités individuelles.

Pour ce faire, nous ferons appel à la démarche de Hsiao (2003) que nous allons appliquer sur notre équation de base $\left(y_{j, t}=\alpha_{j}+\mu_{j} x_{j, t}+\varepsilon_{j, t}\right.$ ), et qui distingue entre quatre (4) configurations comme expliqué par Bourbonnais (2009) $)^{12}$ :

- $1^{\mathrm{er}}$ cas : Les constantes $\alpha_{j}$ et les coefficients $\mu_{j}$ sont tous identiques pour les 31 pays de notre échantillon, ce qui correspond à une homogénéité totale. L'équation de base devienne : $y_{j, t}=\alpha+\mu x_{j, t}+\varepsilon_{j, t}$;

$2^{\text {ème }}$ cas : Les coefficients et les constantes sont tous différents pour l'ensemble des pays de notre échantillon, ce qui correspond à l'hétérogénéité totale, il faut donc rejeter la structure en panel. L'équation dans ce cas est : $y_{j, t}=\alpha_{j}+\mu_{j} x_{j, t}+\varepsilon_{j, t}$

$3^{\text {eme }}$ cas: Les constantes sont toutes identiques, alors que les coefficients sont différents pour chaque pays, de sorte que l'équation est : $y_{j, t}=\alpha+\mu_{j} x_{j, t}+\varepsilon_{j, t}$. Il s'agit d'une hétérogénéité des coefficients des variables explicatives et homogénéité des termes constants ;

$4^{\text {ème }}$ cas : les constantes sont différentes, et les coefficients sont tous identiques. C'est le cas du modèle à effets individuels, et l'équation de base devient :

$$
y_{j, t}=\alpha_{j}+\mu x_{j, t}+\varepsilon_{j, t} .
$$

Ainsi, le choix de la spécification appropriée à notre modèle parmi ces quatre (4) configurations dépend des tests d'homogénéité. A cet effet, il est possible de procéder par un seul test simplifié (celui de Fisher ${ }^{13}$ ) permettant de confronter deux (2) principales hypothèses parmi les quatre (4) configurations expliquées ci-dessus, à savoir ${ }^{14}$ :

\footnotetext{
12 BOURBONNAIS R. (2009), Econométrie, $7^{\mathrm{ème}}$ Editions, Dunod, Paris, P 331.

${ }^{13}$ Il est à préciser que le logiciel Stata donne directement la statistique de ce test avec les résultats de la régression par effets fixes.

${ }^{14}$ ROUS P. (2009), Modèles estimés sur données de panel, Faculté de Droit et des Sciences Economiques de Limoges, Site web : www.unilim.fr/pages_perso/philippe.rous/Donnes_de_Panel.pdf; Consulté le 02/09/2014.
} 
후 L'hypothèse $\mathrm{H}_{0}$ : le modèle qui postule l'homogénéité complète des comportements d'un individu à l'autre, soit l'équation : $y_{j, t}=\alpha+\mu x_{j, t}+$ $\varepsilon_{j, t}$

F L'hypothèse $\mathrm{H}_{1}$ : le modèle qui intègre une hétérogénéité partielle des comportements prenant la forme de constantes spécifiques : $y_{j, t}=\alpha_{j}+$ $\mu x_{j, t}+\varepsilon_{j, t}$.

Les résultats des tests donnent des statistiques de Ficher nettement inférieurs à $1 \%$ pour les deux (2) équations (importations et exportations), ce qui nous ramène à rejeter $\mathrm{H}_{0}$.

En effet, pour le cas des exportations, nous avons obtenu : $\mathrm{F}(30,334)$ $=93,00$ avec Prob $>F=0,0000$;

Pour le cas des importations, nous avons obtenu : $\mathrm{F}(30,336)=75,57$ avec Prob $>\mathrm{F}=0,0000$

A la lecture de ces résultats, il s'avère qu'il existe des effets spécifiques pour les deux (2) équations, ce qui implique que l'utilisation des données de panel est justifiée. Cela nous ramène donc à procéder à l'estimation de nos équations afin de détecter s'il s'agit des effets fixes ou aléatoires.

\section{II.2. Estimation du modèle statique}

Notre étude utilise un cadre de données de panel. L'avantage des données de panel est que les séries chronologiques et la section des observations sont combinées pour augmenter la taille de l'échantillon, afin de donner plus de variabilité et de réduire la multi-colinéarité entre les variables.

D'après les spécifications du modèle obtenues, les équations du modèle statique se présentent, ainsi, comme suit :

- Les exportations 1 du $\operatorname{Maroc}(X)$ :

$$
\begin{aligned}
\operatorname{LnX}_{j t}=\alpha_{0}+ & \beta 1 \text { LnPIB. Ma }_{t}(P P A)+\beta 2 \text { LnPIB }_{j t}(P P A) \\
& +\beta 3 \text { LnDistance }_{j}+\beta 4 \text { LnTCH H }_{j t}+\beta 5 \text { LnDMPE }_{j t} \\
& +\beta 6 \text { ALE }_{j t}+\beta 7 \text { Lang }_{j}+\beta 8 \text { Colon }_{j}+\alpha_{j}+\lambda_{t}+\varepsilon_{j t}
\end{aligned}
$$

- Les importations 1 du $\operatorname{Maroc}(\mathbf{M})$ :

$$
\begin{aligned}
\text { LnM }_{j t}=\alpha_{0} & +\theta 1 \text { LnPIB. Ma }_{t}(P P A)+\theta 2 \text { LnPIB }_{j t}(P P A) \\
& +\theta 3 \text { LnDistance }_{j}+\theta 4 \text { LnTCH }_{j t}+\theta 5 \text { LnDMPM }_{j t} \\
& +\theta 6 \text { ALE }_{j t}+\theta 7 \text { Lang }_{j}+\theta 8 \text { Colon }_{j}+\alpha_{j}+\lambda_{t}+\varepsilon_{j t}
\end{aligned}
$$

avec :

$\mathrm{X}_{\mathrm{jt}}$ : la variable dépendante de l'équation des exportations et qui représente les exportations du Maroc vers le pays $\mathrm{j}$ (partenaire euroméditerranéen), exprimées en Dirham ; 
* $\mathrm{M}_{\mathrm{jt}}$ : la variable dépendante de l'équation des importations et qui représente les importations du Maroc vers le pays partenaire j, exprimées en Dirham ;

PIB.Mat : le revenu du Maroc mesuré par le PIB en parité du pouvoir d'achat par habitant, en Dirham ;

* PIB $_{\mathrm{jt}}$ : le revenu du pays j mesuré par le PIB en parité du pouvoir d'achat par habitant, en Dirham ;

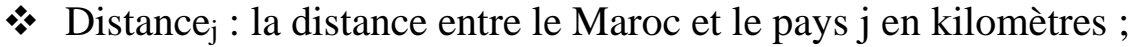

- $\mathrm{TCH}_{\mathrm{j} t}$ : le taux de change réel moyen entre la monnaie du Maroc et celle du pays $\mathrm{j}$ enregistré dans la période $\mathrm{t}$;

* $\mathrm{ALE}_{\mathrm{jt}}$ : une variable muette qui prend la valeur 1 lorsqu'il y a un accord de libre-échange entre le Maroc et le pays $\mathrm{j}$ à la période $t$, alors qu'elle prend la valeur 0 le cas échéant ;

- DMPE $E_{\mathrm{jt}}$ : le droit moyen préférentiel accordé aux exportations du Maroc par le pays j durant l'année t ;

- $\mathrm{DMPM}_{\mathrm{jt}}$ : le droit moyen préférentiel accordé par le Maroc aux importations du pays j durant l'année t ;

* Lang $_{\mathrm{j}}$ : une variable muette qui prend la valeur 1 si le Maroc et le partenaire j partagent une langue commune (l'arabe), et 0 le cas échéant ;

* Colon $n_{\mathrm{j}}$ : une variable muette qui prend la valeur 1 si le pays partenaire $\mathrm{j}$ a eu ou avait un lien de colonisation avec le Maroc, et 0 dans le cas échéant ;

* Les paramètres à estimer : $\beta 1, \beta 2 \ldots$ pour l'équation des exportations et $\theta 1, \theta 2 \ldots$ pour les importations ;

* Des effets spécifiques au pays $\mathrm{j}: \alpha_{\mathrm{j}}$

* Des effets temporels : $\lambda_{t}$

* Les erreurs : $\varepsilon_{\mathrm{jt}}$

\section{II.2.1. Estimation par les effets fixes et les effets aléatoires}

Tout d'abord, il faut noter que lorsque l'estimation se fait avec des effets fixes, les $\alpha_{\mathrm{j}}$ sont considérés comme des facteurs permettant de capter les hétérogénéités inobservables entre les pays de l'échantillon.

La méthode des effets fixes se base sur l'estimateur «within ${ }^{15}$ » qui précise les effets individuels, et qui permet de combler les lacunes de la méthode MCO. Or, l'estimation par les effets fixes ne permet pas d'estimer les variables invariantes dans le temps. En effet, notre modèle, que ce soit pour les exportations ou les importations, contient certaines variables invariantes

\footnotetext{
${ }^{15}$ L'hypothèse du modèle à effets fixes est que les effets individuels $(\alpha j)$ des données sont constants. L'estimateur Within est un estimateur BLUE (Best Linear Unbiased Estimator) ou meilleur estimateur linaire sans biais.
} 
dans le temps, et qui sont essentielles à notre analyse, notamment la variable distance. D'ailleurs, dans l'estimation par effets fixes, les variables stables dans le temps sont omises automatiquement par le logiciel ${ }^{16}$.

Tableau 1 : L'estimation de l'équation des exportations 1 (modèle statique)

\begin{tabular}{|c|c|c|c|c|c|}
\hline \multirow{2}{*}{ Variables } & \multicolumn{2}{|c|}{ Effets fixes } & \multicolumn{2}{|c|}{ Effets aléatoires } & \multirow{2}{*}{ Signe attendu } \\
\hline & coefficients & t-Statistics & coefficients & t-Statistics & \\
\hline PPA Maroc & 0,3106 & 0,56 & 0,7946 & 1,35 & + \\
\hline PPA pays & 1,3835 & $3,55 * * *$ & 1,0256 & $2,49 * * *$ & + \\
\hline Distance & & & $-1,3742$ & $-2,38 * *$ & - \\
\hline Taux de change & $-0,6846$ & $-2,31 * *$ & $-0,7117$ & $-3,52 * * *$ & + \\
\hline DMP d'exportation & $-0,1114$ & $-0,57$ & $-0,0682$ & $-0,32$ & - \\
\hline Accord de libre-échange & 0,6126 & $2,94 * * *$ & 0,7623 & $3,24 * * *$ & + \\
\hline Langue & & & 2,3673 & $2,8 * * *$ & + \\
\hline Colonisateur & & & 3,3701 & $4,51 * * *$ & + \\
\hline Constante & 0,9123 & 0,11 & 10,3151 & 1,13 & \\
\hline R-Sq (within) & \multicolumn{2}{|c|}{0,3217} & \multicolumn{2}{|c|}{$\mathbf{0 , 3 1 8 6}$} & \\
\hline R-Sq (between) & \multicolumn{2}{|c|}{0,0435} & \multicolumn{2}{|c|}{0,3555} & \\
\hline R-Sq (overall) & \multicolumn{2}{|c|}{0,0639} & \multicolumn{2}{|c|}{$\mathbf{0 , 3 4 7 1}$} & \\
\hline Nb. d'observations & \multicolumn{4}{|c|}{370} & \\
\hline F-test / Wald Chi2 & \multicolumn{2}{|c|}{ 13,37 (Prob. : 0,0000) } & \multirow{2}{*}{\multicolumn{2}{|c|}{$\mathbf{1 8 6 , 1 4}$ (Prob. : 0,0000) }} & \\
\hline Hausman test & \multicolumn{3}{|c|}{ 8,23 (Prob. : 0,1442) } & & \\
\hline
\end{tabular}

Note : *** significatif à $1 \% ; * *$ significatif à $5 \% ; *$ significatif à $10 \%$

Tableau 2 : L'estimation de l'équation des importations 1 (modèle statique)

\begin{tabular}{|c|c|c|c|c|c|}
\hline \multirow{2}{*}{ Variables } & \multicolumn{2}{|c|}{ Effets fixes } & \multicolumn{2}{|c|}{ Effets aléatoires } & \multirow{2}{*}{ Signe attendu } \\
\hline & coefficients & t-Statistics & coefficients & t-Statistics & \\
\hline PPA Maroc & $-0,6946$ & $-0,93$ & $-0,6897$ & $-0,93$ & + \\
\hline PPA pays & 1,5936 & $2,13 * *$ & 1,1909 & $2,27 * *$ & + \\
\hline Distance & & & $-0,7851$ & $-1,61$ & - \\
\hline Taux de change & $-1,1964$ & $-2,85 * * *$ & $-1,3793$ & $-4,66 * * *$ & - \\
\hline DMP d'importation & $-0,6432$ & $-1,62$ & $-0,7869$ & $-2,65 * * *$ & - \\
\hline Accord de libre-échange & 0,3066 & 0,70 & 0,4650 & 1,10 & + \\
\hline Langue & & & 1,1957 & 1,06 & + \\
\hline Colonisateur & & & 2,7318 & $4,62 * * *$ & + \\
\hline Constante & 12,9015 & 0,87 & 24,1528 & $2,02 * *$ & \\
\hline R-Sq (within) & \multicolumn{2}{|c|}{0,4637} & \multicolumn{2}{|c|}{0,4603} & \\
\hline R-Sq (between) & \multicolumn{2}{|c|}{0,1622} & \multicolumn{2}{|c|}{0,3981} & \\
\hline R-Sq (overall) & \multicolumn{2}{|c|}{0,2009} & \multicolumn{2}{|c|}{$0,40 z 47$} & \\
\hline Nb. d'observations & \multicolumn{4}{|c|}{372} & \\
\hline
\end{tabular}

${ }^{16}$ C'est l'une des principales limites du modèle des effets fixes, puisqu'il ne permet pas d'estimer directement les variables invariantes dans le temps. Une seconde étape d'estimation de ces variables s'impose, mais étant donné que dans notre cas celles-ci n'incluent pas des variables importantes pour notre étude, nous nous contentons des résultats obtenus avec omission des variables invariantes dans le temps. 
Néanmoins, nous pouvons remarquer que le R2within relatif aux exportations est égale à 0,32 . Ce qui implique que notre modèle gravitationnel estimé avec des effets fixes est satisfaisant pour le cas des exportations. En effet, la faiblesse du R2within dans les modèles transversaux (de panel) en général, et les modèles de gravité en particulier, est très fréquente même avec des statistiques de Ficher «F-test» significatives, ce qui est vrai pour notre cas $^{17}$.

En même temps, les résultats obtenus de l'équation des importations démontrent que l'estimateur des effets fixes donne des coefficients significatifs, à la fois pour les variables PIB en PPA du pays partenaires, le taux de change, le droit moyen préférentiel aux importations, ainsi que l'accord de libre-échange, et les signes prévus correspondent aux signes obtenus. Or, nous constatons que la variable PIB en PPA du Maroc n'est pas significative statistiquement, et son signe ne correspond pas au signe prévu, et par conséquent cette variable n'a aucun impact sur les flux des importations.

De même, nous constatons que le R2within est équivalent à 0,46 , ce qui implique que les résultats d'estimation des effets fixes de l'équation des importations sont satisfaisants.

A ce niveau, nous pouvons considérer que les résultats obtenus par l'estimation avec les effets fixes sont consistants et efficaces. Mais puisque le modèle à effets fixes n'intègre pas les variables invariantes dans le temps. Donc, dans le modèle à effets fixes, les effets des variables constantes ne peuvent pas être identifiés et estimés d'une manière simple. Ainsi, nous allons adopter une régression avec l'estimateur des effets aléatoires «between », qui utilise la méthode des moindres carrés généralisés (MCG), afin de pouvoir intégrer même les variables constantes dans la régression et les coefficients associés peuvent être estimés.

Les résultats obtenus par la méthode MCG sont représentés dans le tableau 9 et le tableau 10 respectivement pour l'équation des « exportations $1 »$ et des « importations $1 »$.

Nous remarquons ainsi, d'après le tableau 9, que tous les signes obtenus des coefficients des variables relatives aux « exportations $1 »$ ont les signes attendus, sauf la variable taux de change. Alors que les variables PIB

${ }^{17}$ Cette remarque a été relevée dans la majorité des études se basant sur des modèles de gravité, comme c'est le cas de l'analyse de Amr Sadek Hosny (Does GAFTA Defy Gravity ? ; publiée dans l'Euro Journals, Middle Eastern Finance and Economics, 2011), qui précise qu'à la différence des séries temporelles pouvant résulter des $\mathrm{R}_{2}$ proches de 1 , les modèles transversaux donnent, le plus souvent des $\mathrm{R}_{2}$ faibles. 
en PPA du pays partenaire, accord de libre-échange, langue, liens de colonisation, ainsi que distance sont statistiquement significatifs respectivement au seuil de $1 \%$ et $5 \%$. D'où l'influence de ces variables sur les flux des exportations.

Alors que nous remarquons que pour les résultats du tableau 10 relatifs à l'équation des « importations1 », que tous les signes obtenus des coefficients des variables correspondent aux signes prévus, sauf le PIB en PPA du Maroc. Tous les coefficients de ces variables sont significatifs au seuil de 1\%, et la variable des liens coloniaux est significative au seuil de $5 \%$, et ont par conséquent un impact sur les flux des importations.

En fait, l'estimateur «between » avec la méthode MCG nous offre des résultats satisfaisants, avec un $\mathrm{R} 2$ between $=0,35$ pour le cas des exportations et un R2between $=0,39$ pour l'équation des importations.

Par ailleurs, il est nécessaire de procéder par le test d'«Hausman» qui nous permettra de vérifier la pertinence du choix entre le modèle à effets fixes et le modèle à effets aléatoires, et faciliter donc la détection d'endogénéité.

En fait, dans ce test, l'hypothèse évaluée concerne l'existence d'une corrélation des effets individuels et des variables explicatives.

Et comme nous pouvons le constater dans les résultats du test ci-dessus (tableaux 9 et 10), le test d'Hausman montre qu'il ne faut pas rejeter l'hypothèse nulle selon laquelle il n'ya pas de mauvaise spécification du modèle à effets aléatoires au niveau de 5\%.

Autrement dit, le modèle à effets aléatoires est plus approprié pour les données par rapport à l'équation des exportations et des importations. En conséquence, nous allons poursuivre les estimations en introduisant une dimension dynamique à l'analyse des effets des accords de libre-échange sur les échanges commerciaux du Maroc. C'est ainsi que nous allons faire appel au modèle dynamique à la fois pour l'équation des exportations et celle des importations.

En effet, le modèle statique nous a permis d'obtenir des résultats assez fiables en introduisant des variables instrumentales dans le cadre de la Méthode des Moindres Carrés Généralisés. Cependant, il ne prend pas en considération l'effet retardé des variables dépendantes. Par contre, le modèle dynamique nous permettra de tester sur nos deux (2) équations l'impact d'introduction des variables dépendantes retardées sur la fiabilité des résultats et d'apprécier, de ce fait, les changements qu'ont subis les flux des échanges commerciaux entre le Maroc et ses partenaires euro-méditerranéens.

\section{II.2.2. Estimation du modèle dynamique}

Il est distingué dans la littérature entre statique et dynamique des modèles de données de panel. Le modèle statique de données de panel comprend les effets fixes et la méthode à effets aléatoires, tandis que les 
modèles dynamiques sur données de panel sont ceux qui incluent une variable dépendante retardée comme variable explicative.

Notre étude reposera sur un modèle en panel dynamique, qui tient compte du retard de la variable endogène. L'adoption d'une spécification dynamique en prenant en considération ces retards est sensée déceler l'influence de l'adoption des accords de libre-échange sur les flux commerciaux du Maroc.

Autrement dit, nous pensons que le modèle dynamique nous permettra d'observer l'évolution des échanges commerciaux dans le cadre des accords de libre-échange conclus par le Maroc dans l'espace euro-méditerranéen, tant que le modèle dynamique permet de prendre en considération l'influence des retards sur les échanges du pays.

En effet, nos équations (exportations 1 et importations 1) dans le modèle statique ont été étudiées dans le cadre d'une analyse constante. Or, il est avéré que le volume des échanges commerciaux entre deux (2) pays pour une année donnée est fortement corrélé avec le volume de l'année précédente en raison de la relative stabilité des facteurs qui facilitent l'adéquation de la production aux échanges commerciaux, tels que les sources exogènes d'avantages comparatifs ou encore les niveaux d'industrialisation. Les équations (exportations 2 et importations 2 présentées ci-dessous) doivent donc être formulées de manière dynamique. Pour ce faire, il suffit d'ajouter au modèle, la variable dépendante retardée d'une période comme proposé par Egger (2001). Les équations auront alors la forme log-linéaire suivante :

- Les exportations 2 du $\operatorname{Maroc}(X)$ :

$$
\begin{aligned}
& \operatorname{Ln} X_{j t}=\alpha_{0}+\beta 1 \operatorname{Ln} X_{j t-1}+\beta 2 \operatorname{LnPIB.} \operatorname{Ma}_{t}(P P A) \\
& +\beta 3 \text { LnPIB }_{j t}(P P A)+\beta 4 \text { LnDistance }_{j}+\beta 5 \text { LnTCH }_{j t} \\
& +\beta 6 L_{n D M P E}+\beta 7 \text { ALE }_{j t}+\beta 8 \text { Lang }_{j}+\beta 9 \text { Colon }_{j} \\
& +\alpha_{j}+\lambda_{t}+\varepsilon_{j t}
\end{aligned}
$$

- Les importations 2 du Maroc (M) :

$$
\begin{aligned}
\text { LnM }_{j t}=\alpha_{0} & +\theta 1 \text { LnM }_{j t-1}+\theta 2 \text { LnPIB. Ma }_{t}(P P A) \\
& +\theta 3 \text { LnPIB }_{j t}(P P A)+\theta 4 \text { LnDistance }_{j} \\
& +\theta 5 \text { LnTCH }_{j t}+\theta 6 \text { LnDMPM }_{j t}+\theta 7 \text { ALE }_{j t} \\
& +\theta 8 \text { Lang }_{j}+\theta 9 \text { Colon }_{j}+\alpha_{j}+\lambda_{t}+\varepsilon_{j t}
\end{aligned}
$$

avec :

* $\mathrm{X}_{\mathrm{jt}-1}$ : le volume des exportations du Maroc vers le pays $\mathrm{j}$ (pays partenaire) retardé d'une année ;

- $\mathrm{M}_{\mathrm{jt}-1}$ : le volume des importations du Maroc vers le pays j à la date t-1. 
Nous avons pris en considération le retard, afin d'éviter les problèmes d'auto corrélation, qui peuvent résulter d'autres mesures d'ordre commerciales, fiscales ou autres, qui influencent le volume des échanges bilatéraux, et qui ne sont pas représentés par des variables dans le modèle de gravité (Bair et Bergstand (2007).

Ainsi, nous allons associer deux (2) tests à l'estimateur de la méthode des moments généralisée (MMG ou GMM en anglais) en panels dynamiques :

- le test de sur-identification de Sargan/Hansen qui permet de tester la validité des variables retardées comme instruments, et repose essentiellement sur l'hypothèse nulle suivante ( $\mathrm{H} 0$ : les instruments sont valides);

- le test d'autocorrélation d'Arellano Bond, où l'hypothèse nulle est l'absence d'autocorrélation des erreurs de l'équation en différence, de sorte que (H0 : Absence de corrélation des résidus).

Nous estimons donc les équations de gravité dynamiques relatives aux exportations et aux importations par la méthode MMG en système en deux (2) étapes, puisque tous nos panels sont cylindrés. Les résultats de nos estimations sont présentés dans le tableau 11 pour les «exportations 2 » et le tableau 12 pour les «importations $2 »$. Nous avons retenu au plus 5\% pour les seuils de significativité des estimations. Ainsi les résultats de l'estimation du modèle dynamique se présentent ainsi :

Tableau 3 : L'estimation de l'équation des exportations 2 par la méthode MMG

\begin{tabular}{|c|c|c|c|}
\hline \multirow{2}{*}{ Variables } & \multicolumn{2}{|c|}{ GMM } & \multirow{2}{*}{ Signe attendu } \\
\hline & coefficients & t-statistics & \\
\hline $\operatorname{Exp}_{t-1}$ & 0,795 & $26,32 * * *$ & + \\
\hline PPA Maroc & 0,870 & $7,15 * * *$ & + \\
\hline PPA pays & $-0,164$ & $-2,70 * *$ & + \\
\hline Distance & 0,360 & 1,19 & - \\
\hline Taux de change & 0,369 & 1,36 & + \\
\hline DMP d'exportation & $-0,511$ & $-12,47 * * *$ & - \\
\hline Accord de libre-échange & 0,591 & $6,68 * * *$ & + \\
\hline Langue & $-0,636$ & $-1,19$ & + \\
\hline Colonisateur & 3,313 & $2,00 * *$ & + \\
\hline Constante & $-8,009$ & $-2,68 * *$ & \\
\hline Nombre d'observations & \multicolumn{3}{|c|}{338} \\
\hline Nombre d'instruments & \multicolumn{3}{|c|}{93} \\
\hline Nombre de groupe & \multicolumn{3}{|c|}{31} \\
\hline F-test & \multicolumn{3}{|c|}{ 1139,60 (Prob. : 0,000) } \\
\hline AR (1) & \multicolumn{3}{|c|}{$-2,51$ (Prob. : 0,012) } \\
\hline $\mathrm{AR}(2)$ & \multicolumn{3}{|c|}{$-0,38$ (Prob.: 0,705) } \\
\hline Test de Sargan & \multicolumn{3}{|c|}{ 280,64 (Prob. : 0,000) } \\
\hline
\end{tabular}




\begin{tabular}{|c|r|}
\hline Test de Hansen & 25,87 (Prob. : 1,000) \\
\hline
\end{tabular}

Note : *** significatif à $1 \%$; ** significatif à $5 \%$; * significatif à $10 \%$

Nous remarquons d'après les résultats de l'estimation de l'équation des exportations 2 , que l'hypothèse nulle d'absence d'autocorrélation de premier ordre AR(1) est rejetée au seuil de significativité de 5\%, alors que l'hypothèse nulle d'absence d'autocorrélation de second ordre AR(2) ne peut pas être rejetée. Dès lors les spécifications de notre modèle sont valides, selon Arellano et Bond (1991).

Par ailleurs, le test de sur-identification de Hansen J-test (Prob. = 1,000) ne rejette pas l'hypothèse nulle de validité des variables retardées choisies, pour notre modèle comme instruments valides. Nous acceptons, donc, la spécification conservée dans le modèle et la validité de tous les instruments utilisés.

Donc, d'après les différents tests statistiques qui ont été menés, nous pouvons dire qu'il y a suffisamment de preuves pour conclure que les tests statistiques en question, valident les principales hypothèses d'estimation par la méthode de MMG en système, et que ce modèle est un mécanisme de génération des statistiques appropriées.

Nous constatons également que les résultats de l'estimation nous indiquent que la variable endogène retardée $\left(\operatorname{Exp}_{\mathrm{t}-1}\right)$ a un impact fortement positif et significatif sur les exportations. D'où l'intérêt de raisonner en termes d'équation de gravité dynamique.

Nous remarquons aussi, pour notre variable indépendante d'intérêt (ALE), que l'adoption des accords de libre-échange avec les pays méditerranéens influence notablement le flux des exportations du Maroc, étant donné que le coefficient de l'accord de libre-échange demeure significatif et correspond au signe prévu. Ceci indique que l'entrée en vigueur des accords de libre-échange avec les pays méditerranéens stimule davantage les flux des exportations vers ces pays. Ceci d'ailleurs nous semble évident, mais il convient de se demander si c'est le cas pour les importations.

L'adoption des accords de libre-échange, permettra certainement au Maroc de bénéficier des droits moyens préférentiels aux exportations. En revanche, nous remarquons, d'après les résultats de l'estimation, que le coefficient de la variable «LnDMPE » est significatif et correspond au signe prévu. De ce fait, l'obtention des droits moyens préférentiels aux exportations a certainement un effet sur les exportations. Donc, la conclusion des accords de libre-échange favorise davantage l'augmentation des flux des exportations nationales.

Certainement, la distance est une variable gravitationnelle importante, puisque tant qu'elle est grande, elle bloque les flux commerciaux. Donc elle est négativement liée au commerce bilatéral entre les partenaires. En revanche, 
dans notre cas, le signe du coefficient de la variable distance ne correspond pas au signe prévu, ni même significatif, ce qui signifie que la distance entre le Maroc et ses partenaires commerciaux méditerranéens n'influence pas les flux de ses exportations. D'ailleurs, nous pouvons remarquer que les relations commerciales entre le Maroc et l'Algérie n'ont pas abouties à la conclusion d'un accord de libre-échange, malgré le partage des frontières. Le Maroc par ailleurs, s'est orienté vers la Turquie et la Jordanie en négligeant l'impact de la distance et les coûts de transport.

L'estimation démontre clairement que les exportations du Maroc sont une fonction croissante de la taille de l'économie, puisque le coefficient associé à la variable PIB en PPA du Maroc est positif et significatif.

Tandis que le signe du coefficient relatif au PIB en PPA du pays partenaire, ne correspond pas au signe prévu, et pourtant il se trouve qu'il est significatif. Ceci dit que la taille de l'économie du pays partenaire a une influence sur les flux des exportations marocaines. Autrement dit, les écarts de développement jouent un rôle important dans ce cas.

En même temps, notons que les liens de colonisation et historiques, précisément avec l'Espagne et la France, ont une influence déterminante sur les exportations nationales étant donné que le signe obtenu du coefficient de la variable «Colon» correspond au signe prévu et est significatif.

Nous constatons aussi que le coefficient de la variable «Lang », qui représente le partage d'une langue commune dans notre étude, à savoir, la langue arabe, a un signe négatif et n'est pas significatif. Ce qui indique que le partage d'une langue commune précisément avec les pays arabes, avec lesquels le Maroc a conclu des accords de libre-échange (Egypte, Jordanie, Tunisie) n'a aucune influence sur les exportations marocaines.

Tableau 4 : L'estimation de l'équation des importations 2 par la méthode MMG

\begin{tabular}{|c|c|c|c|}
\hline \multirow{2}{*}{ Variables } & \multicolumn{2}{|c|}{ GMM } & \multirow{2}{*}{ Signe attendu } \\
\hline & coefficients & t-statistics & \\
\hline IMP $_{t-1}$ & 0,636 & $5,08 * * *$ & + \\
\hline PPA Maroc & $-0,562$ & $-0,76$ & + \\
\hline PPA pays & $-0,392$ & $-1,51$ & + \\
\hline Distance & $-1,080$ & $-1,41$ & - \\
\hline Taux de change & $-0,644$ & $-2,25 * *$ & - \\
\hline DMP d'importation & $-0,427$ & $-2,36 * *$ & - \\
\hline Accord de libre-échange & 0,955 & $2,88 * * *$ & + \\
\hline Langue & $-1,212$ & $-2,46 * *$ & + \\
\hline Colonisateur & 2,494 & 1,18 & + \\
\hline Constante & 28,222 & $2,95 * * *$ & \\
\hline Nombre d'observations & \multicolumn{3}{|c|}{341} \\
\hline Nombre d'instruments & \multicolumn{3}{|c|}{88} \\
\hline Nombre de groupe & \multicolumn{3}{|c|}{31} \\
\hline F-test & \multicolumn{3}{|c|}{ 88,25 (Prob. : 0,000) } \\
\hline
\end{tabular}




\begin{tabular}{|c|c|}
\hline AR (1) & $-1,99$ (Prob. : 0,046) \\
\hline AR (2) & $-1,13$ (Prob.: 0,256) \\
\hline Test de Sargan & $291,60($ Prob. : 0,000) \\
\hline Test de Hansen & $28,00($ Prob. : 1,000$)$ \\
\hline
\end{tabular}

Note : *** significatif à $1 \% ; * *$ significatif à $5 \% ; *$ significatif à $10 \%$

Comme pour notre précédente estimation, nous constatons que, les hypothèses d'Arellano et Bond sont vérifiée. L'hypothèse nulle d'absence d'autocorrélation du premier ordre AR(1) est rejetée au seuil de significativité de 5\%, alors que l'hypothèse nulle d'absence d'autocorrélation du second ordre $\operatorname{AR}(2)$ ne peut pas être rejetée.

De même, le test de sur-identification de Hansen J-test (Prob.= 1,000) ne rejette pas l'hypothèse de validité des variables retardées comme instruments dans notre modèle.

Comme nous pouvons le remarquer dans notre modèle, le nombre d'instruments ne dépasse pas le nombre d'observations ( 88 instruments < 341 observations). Même le signe de la statistique de Hansen-J est parfait avec une p-value égale à 1,00. Il est à rappeler également que Roodman (2006) et Bond (2002) suggèrent que le coefficient de la variable dépendante retardée, soit inférieur à 1 , ce qui est affirmé par notre équation (importations 2$)^{18}$.

De même, nous remarquons que pour tous les pays de notre étude, la variable dépendante retardée d'une période est significative et positive, ce qui justifie la nécessité du raisonnement en termes d'équation de gravité dynamique même pour le cas des importations.

Les résultats concernant les droits moyens préférentiels aux importations, et la variable relative à l'adoption des accords de libre-échange, sont significatifs et correspondent à nos attentes. Ce résultat implique que la conclusion d'un accord de libre-échange, et l'octroi de droits moyens préférentiels aux importations, encouragent le Maroc à importer davantage de ses partenaires méditerranéens avec lesquels il a signé des accords de libreéchange, ce qui explique la forte croissance des importations nationales.

Alors que la variable gravitationnelle représentée par la distance «Lndist »n'est pas significative, ce qui implique que la distance n'influence pas le flux des importations du Maroc, qui émanent de ses partenaires méditerranéens.

Tandis que, la variable qui représente la compétitivité de l'économie nationale, à savoir «le taux de change », est conforme à nos attentes et significative. Ainsi, nous remarquons que l'appréciation du Dirham contribue davantage à l'augmentation des flux des importations du Maroc.

\footnotetext{
${ }^{18}$ En effet, l'estimateur MCO donne un coefficient de la variable exportation retardée de 0,88 . L'estimateur des effets fixes, quant à lui, donne un coefficient de 0,43 . Ce qui signifie que le coefficient obtenu par l'estimateur MMG est compris entre les deux : $0,43<\mathbf{0 , 6 3}<0,88$.
} 
Par contre, il est à noter que la taille de l'économie marocaine, ainsi que celle des pays partenaires méditerranéens n'a pas d'influence sur le flux des importations nationales, puisque les coefficients des variables «PIB en PPA du Maroc» et le «PIB en PPA du pays partenaires» ne sont pas significatifs et n'ont pas les signes prévus.

Il était prévu que les liens de colonisation favorisent les flux des importations marocaines qui émanent de ces pays. Les résultats obtenus ressortent par contre contradictoires à ces résultats prévus. Les liens historiques et de colonisations, plus précisément avec l'Espagne et la France, ont, ainsi, un impact sur les importations marocaines. L'élargissement de l'UE a contribué favorablement à la diversification des fournisseurs du pays. Par conséquent, le Maroc ne dépend pas uniquement de ses pays colonisateurs en tant que principaux fournisseurs.

Par contre les résultats montrent que la variable «Lang» est significative. Le signe de son coefficient ne correspond par contre pas aux prévisions. Cela peut être expliqué par le fait que les pays arabes ne sont pas les principaux partenaires commerciaux du Maroc.

\section{Interprétation des résultats obtenus}

Nous pouvons enfin interpréter ce modèle gravitationnel en utilisant les résultats obtenus des effets aléatoires, ainsi que les résultats de l'estimateur MMG en nous penchant sur leurs conséquences.

\section{III.1. Portée de la dimension dynamique dans le modèle}

Un signe significatif et positif des coefficients des variables (Exp t-1) et (Imp t-1) indique que la croissance des exportations et des importations marocaines de l'année précédente a un effet positif sur les performances des exportations et des importations de l'année courante.

Ce résultat nous semble vérifiable dans la réalité, étant donné que les relations commerciales nouvellement établies avec des acteurs étrangers constituent une base pour les entreprises marocaines pour élargir leurs activités d'exportation et d'importation l'année suivante.

En fait, les investissements initiaux ou les coûts irrécupérables supportés par les exportateurs pour établir de nouveaux réseaux de distribution et de services, génèrent souvent la persistance dans les marchandises exportées par des habitudes de consommation et les canaux de distribution nouvellement créées dans le marché étranger. En conséquence, les résultats des importations et des exportations réalisées l'année précédente, fournissent une assise pour les activités des exportations et des importations de l'année en cours.

De ce fait, le modèle en panel dynamique donne une estimation efficiente de notre modèle, comme il nous offre une bonne représentation de la réalité et capture les éléments, qui nous intéressent: les avantages 
économiques que tire le Maroc des accords de libre-échange signés avec les pays méditerranéens. Il s'agit principalement d'une augmentation des flux commerciaux, sur la base de la libéralisation des échanges avec ces pays, qui partagent les mêmes avantages procurés par le positionnement géographique et historique du bassin méditerranéen, et aussi avec toutes les disparités au niveau du développement économique et social entre le Maroc et ces pays.

\section{III.2. Impact significatif des variables d'ouverture commerciale}

La variable relative à la création d'une zone de libre-échange représente l'idée selon laquelle la formation des zones de libre-échange entre le Maroc et ses partenaires commerciaux méditerranéens permet d'intensifier les relations commerciales entre eux. Donc, ces accords de libre-échange ont un impact plus ou moins important sur les flux commerciaux analysés.

En fait, quelque soit l'importance des accords de libre-échange entre le Maroc et ses partenaires commerciaux méditerranéens, ces accords ont contribué à accroitre les importations du Maroc qui émanent de ces pays plus que ses exportations. Cette augmentation est manifestée surtout par le déficit fréquent de la balance commerciale nationale. Par conséquent, on conclut que le Maroc constitue un véritable débouché pour ces pays. De ce fait, les accords de libre-échange qu'a conclu le Maroc avec ces pays ne sont pas donc bénéfiques.

Les résultats des estimations du modèle de gravité montrent également que l'existence des accords de libre-échange, comme première étape du processus d'intégration permettant au Maroc de bénéficier des droits moyens préférentiels aux exportations et aux importations, est de nature à rapprocher l'économie marocaine de ses partenaires, et de stimuler éventuellement les échanges en réduisant les coûts de transactions. En théorie, toute chose étant égale par ailleurs, les pays signataires d'un accord préférentiel de commerce tendent à échanger environ 2,4 fois plus par rapport à des pays qui ne sont engagés dans aucun accord d'intégration commerciale ${ }^{19}$. C'est de cette façon qu'il peut être lu l'effet significatif de la variable relative aux droits moyens préférentiels aux exportations et aux importations, dont le pouvoir explicatif s'est amélioré en passant du modèle statique au modèle dynamique. D'où le fort impact de ces variables sur les flux commerciaux du Maroc vers ses partenaires méditerranéens, et conduit alors à une création significative du commerce.

\footnotetext{
${ }^{19}$ NGOWATTANA S (2005), Effets gravitationnels de l'AFTA sur l'ASEAN : analyse de panel, In Cahiers de recherche de l'équipe universitaire de recherche «Institutions : coordination, organisation », Université Paris Dauphine, N²005-10, P 16.
} 
Graphique 1 : Evolution des droits moyens préférentiels accordés aux exportations marocaines

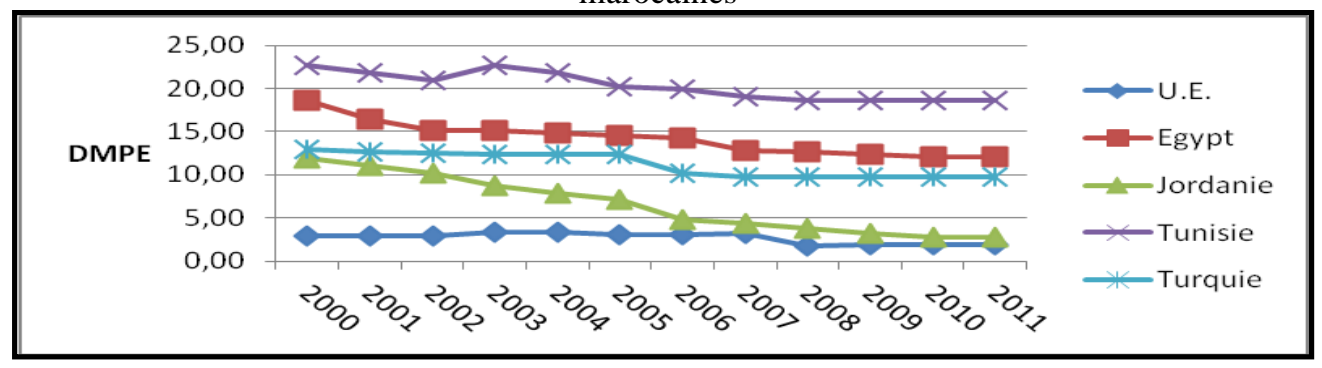

Source : CNCE (Maroc) et WITS (Banque mondiale), 2012

Selon le graphique, il est constaté que les droits moyens préférentiels aux exportations ont certainement diminué, mais leur diminution n'est pas très remarquable tel est le cas des droits moyens préférentiels aux importations comme le montre le graphique ci-dessous.

Graphique 2 : Evolution des droits moyens préférentiels accordés aux importations

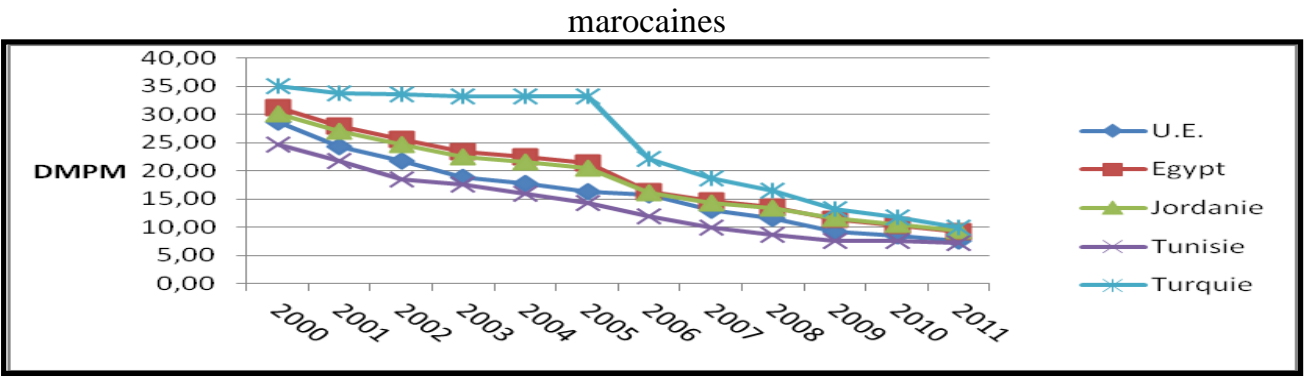

Source : CNCE (Maroc) et WITS (Banque mondiale), 2012

\section{III.3. Influence des variables coloniales et de langue}

Les liens coloniaux ont aussi tendance à faciliter les échanges entre certains pays et le plus souvent positivement. Dans notre étude, les liens de colonisation existants entre le Maroc et ses partenaires commerciaux favorisent aussi le flux des exportations entre eux (Espagne 30,61 milliards de DH en 2011, France 33,17 milliards de DH en 2011). Donc ces pays colonisateurs pourraient être amenés à privilégier les relations commerciales avec le Maroc. Ils pourraient aussi avoir une facilitation d'accès aux ressources de la part du Maroc même après l'indépendance de ce dernier.

De ce fait, l'estimation révèle aussi que le poids de l'histoire joue un rôle significatif dans la détermination de la direction des flux des exportations. En effet, toute chose étant égale par ailleurs, un pays tend à échanger en moyenne 3,34 fois plus avec son colonisateur qu'avec les autres pays ${ }^{20}$.

Graphique 3 : Les parts des exportations du Maroc vers ses partenaires méditerranéens en distinguant ses ex-colonisateurs des pays arabes et du reste de l'Europe (en millions de DH)

${ }^{20}$ Ibidem. 


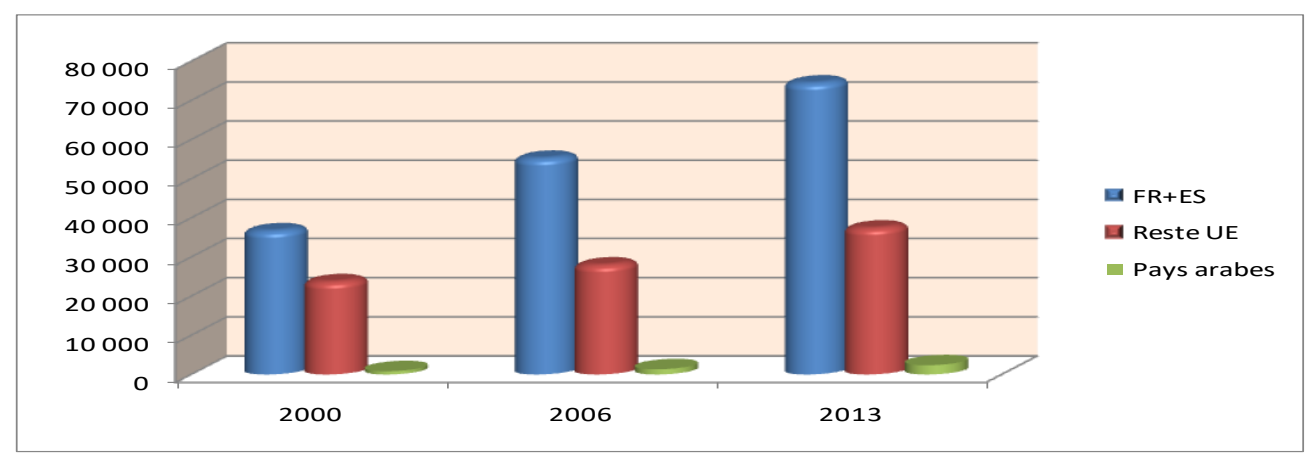

Source : Office des changes et Eurostat, 2014

Les résultats montrent que les liens de colonisation ne favorisent pas nécessairement les importations du Maroc. Ce qui implique que le Maroc n'importe pas forcement de l'Espagne et de la France.

Ce résultat peut être expliqué par la nature des produits importés par le Maroc. En fait, la structure des importations marocaines se caractérise par la prédominance des produits énergétiques.

D'ailleurs, le Maroc est ressorti, sur le mois de janvier 2013, avec une facture énergétique nette de 6,492 milliards de dirhams, en hausse de 5,5\% par rapport à la même période en $2012^{21}$. Il est noté en fait, que le Maroc est confronté à une contrainte énergétique due à sa forte dépendance (plus que $96 \%$ ) vis à vis de l'extérieur, et à l'utilisation massive des énergies traditionnelles dont la biomasse. Cette situation accentue les relations du Maroc avec certains pays arabes et africains, tels que 1'Arabie Saoudite (pétrole), l'Irak, l'Iran, Nigeria, l'Afrique du Sud (charbon), afin de répondre à ses besoins énergétiques, et minimise en termes relatifs la relation du Maroc avec ses ex-colonisateurs.

En ce qui concerne le partage d'une langue commune, cette dernière ne semble pas être un facteur positif sur les importations et même sur les exportations puisque cette variable a perdu sa significativité en allant d'une analyse statique à une analyse dynamique dans l'équation des exportations et son signe se trouve négatif dans l'équation des importations. Mais la définition que nous avions choisie pour la variable langue commune avec les pays arabes peut expliquer ce résultat. Nous avons considéré que le Maroc peut favoriser, dans ses relations commerciales, les pays avec lesquels il partage une langue commune, à savoir « la langue Arabe », alors que l'Anglais et le Français sont les plus utilisées par ces pays. Puisque, en dehors de la Tunisie, la plupart des pays du Moyen Orient de notre échantillon ayant eu des liens historiques avec la Grande Bretagne et la France, ont adopté l'anglais et le français a côté de

${ }^{21}$ Les dernières statistiques énergétiques en date de la Direction de l'observation et de la programmation, relevant du ministère de l'énergie, des mines, de l'eau et de l'environnement. 
l'Arabe (Egypte, Jordanie). Ce qui rend l'Anglais la langue la plus utilisée par la grande majorité de notre échantillon, même par des pays qui n'ont pas été colonisés par la Grande Bretagne. Donc, si la langue commune était limitée à la définition la plus stricte, on devrait s'attendre à l'existence d'une colinéarité du fait de la coexistence, dans une même équation, de cette variable avec la variable passé colonial.

D'ailleurs, ce résultat est reflété dans la réalité, puisque les échanges commerciaux du Maroc avec les pays arabes méditerranéens sont trop limités par rapport à ses échanges avec les pays de l'UE. Ce qui confirme encore une fois la forte dépendance du Maroc vis-à-vis de l’UE.

\section{III.4. Effet des variables gravitationnelles}

Les résultats de notre étude montrent que la distance est une variable non significative et négativement liée à la conclusion des accords de libreéchange entre le Maroc et ses partenaires méditerranéens.

Le signe négatif indique que la création des zones de libre-échange par Maroc est plus solide avec les pays qui sont plus proche géographiquement. Ce résultat est conforme à l'attente, qui confirme que plus la distance est longue, plus elle décourage la création des zones de libre-échange. Ce qui est conforme avec la théorie de Balassa (1986), qui a fait valoir que la zone de libre-échange aura tendance à être plus importante lorsque les pays commerçants sont géographiquement proches les uns des autres.

La principale explication à ce résultat, est que le Maroc a tendance à faire plus de commerce avec les pays, qui sont plus proches de lui géographiquement précisément l'UE, Tunisie, qu'avec d'autres pays qui sont géographiquement plus loin (L’Egypte, la Turquie, la Jordanie).

Pourtant, le Maroc a pris le risque de s'orienter vers ces pays malgré la distance qui le sépare de ces derniers. Nous avons pensé que le Maroc cherchait à travers ces accords à créer une certaine complémentarité avec ces pays. Or, les résultats ne correspondent pas à nos attentes, puisque ses relations commerciales avec ces pays sont plus marquées par la forte concurrence.

D'ailleurs, actuellement nous remarquons que les pays tentent de dépasser la contrainte de distance et des frontières communes, en optant pour une forme d'intégration avec des pays loin géographiquement. En fait, les flux commerciaux se trouvent dynamiques dans ces régions malgré la distance qui peut les séparer. Ce constat est également pris en considération par le Maroc dans le cadre de ses relations avec les pays de l'espace euro-méditerranéen.

Concernant la variable «PIB en PPA du Maroc», celle-ci est jugée statistiquement significative à $1 \%$ et positivement liée aux flux des exportations. Ceci implique que le PIB en PPA du Maroc contribue positivement à accroître les exportations du pays. Une hausse de 1\% du PIB 
en PPA du Maroc entraine, toutes choses égales par ailleurs, une hausse de ses exportations de $0,87 \%$.

Ceci dit, qu'avec l'élargissement de l'économie nationale, le Maroc développe davantage sa capacité de production des biens dont pour lesquels il dispose d'un avantage comparatif, et qui le différencient par rapport à ses concurrents, en facilitant ainsi l'augmentation de ses exportations vers ses partenaires euro-méditerranéens.

Cependant, malgré les efforts fournis par le Maroc pour promouvoir ses exportations, sauf que la forte concurrence avec certains partenaires de l'espace euro-méditerranéen, à savoir la Tunisie, la Turquie, creuse le déficit commercial national, même si le pays est doté d'un avantage comparatif des produits qu'il exporte. Ce constat, s'est manifesté surtout par l'une des plus graves crises de textile, car le commerce international du textile et de l'habillement était régi par l'accord multifibres, en vertu des règles de l'OMC avant le 1er janvier 2005.

Cet accord soumettait les exportations asiatiques, notamment de Chine et d'Inde, à un quota d'exportation vers le marché européen. Cette situation profitait surtout aux pays ayant des accords d'association avec l'UE, comme le Maroc, la Turquie, la Tunisie et les pays de l'Est européen. Et avec la fin de cet accord, il n'y a plus de limites pour les exportations asiatiques. D'où la rude concurrence dans laquelle se trouve le Maroc sur le marché européen sachant que l'UE est le premier partenaire commercial du Maroc. Cette situation a creusé l'augmentation des flux des importations du Maroc et une forte diminution de ses exportations, même si le Maroc confrontait la concurrence des autres pays de l'espace euro-méditerranéen sur ce marché, notamment la Tunisie et la Turquie.

Cependant, cette variable n'a aucune influence sur les flux des importations, puisqu'elle n'est pas significative. Ce qui implique que le flux des importations ne contribue pas à l'élargissement de la taille de l'économie. Ceci parait évident, car malgré le processus d'ouverture choisi par le Maroc depuis le début des années 80 , il se trouve que la balance commerciale du Maroc a toujours présenté un déficit structurel. En fait, ce déficit se creuse davantage depuis 2003 suite à plusieurs facteurs structurels, conjoncturels d'ordre interne et externe, et que le taux de couverture des importations par les exportations a certainement chuté surtout récemment $(45,6 \%$ en 2012 contre $50,5 \%$ à fin février 2011), et pourtant le déficit commercial du Maroc s'établit en hausse de 27,6\% à -32,7 milliards de dirhams à fin février 2012 contre $-25,6$ milliards de dirhams à la même période en 2011, soit une aggravation de 7 milliard de dirhams ${ }^{22}$.

${ }^{22}$ Données de l'Office des changes du Maroc 2011. 
Généralement, les échanges commerciaux du Maroc effectués dans le cadre des accords de libre-échange avec les pays méditerranéens, restent prédominés par les importations. Ce déficit se creuse en fait surtout avec le décalage de croissance économique entre l'économie marocaine et celle de la zone Euro.

En ce qui concerne la variable «PIB en PPA du pays partenaire », nous remarquons qu'elle est statiquement significative pour les exportations et non significative pour les importations. Nous pouvons dire que le Maroc exporte vers ses partenaires méditerranéens, mais ses exportations se focalisent spécialement sur les secteurs concernés par les accords de libre-échange, alors que les importations des pays de notre échantillon reposent essentiellement sur les produits pétroliers, ce qui favorise l'orientation de ces pays vers d'autres fournisseurs autres que le Maroc, à leur tête vient la Chine, la Russie, et les Etats Unis. D'où le signe négatif de cette variable.

Graphique 4 : Evolution des principaux produits à l'exportation 2007-2011

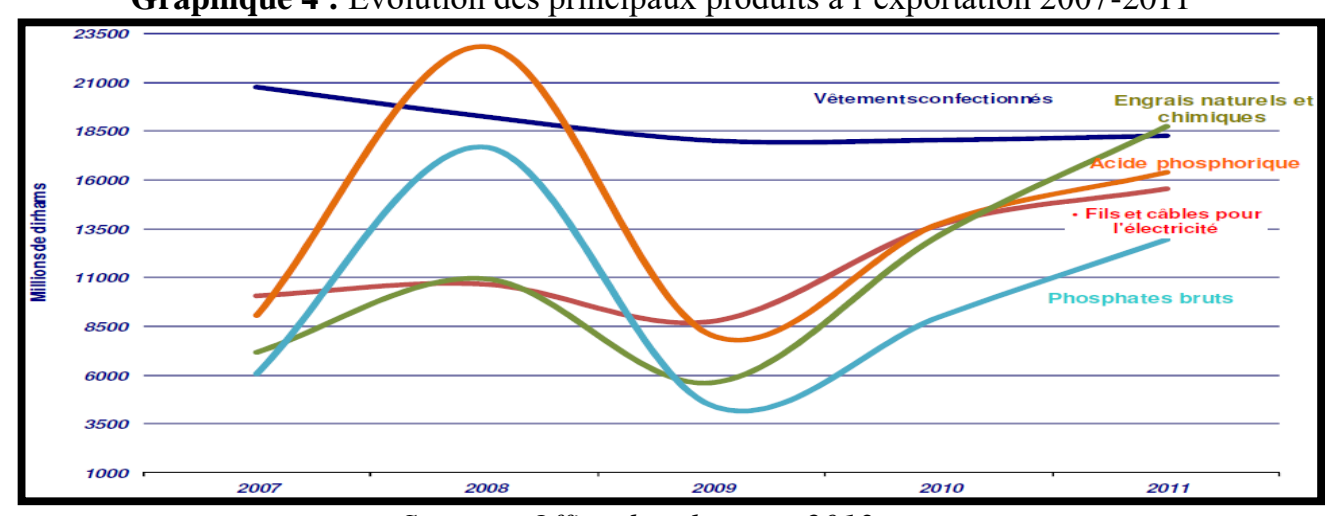

Source : Office des changes, 2013

En fait, les échanges commerciaux du Maroc avec l'extérieur sont largement prédominés par l'Europe, principal partenaire commercial du Maroc. La structure des importations du Maroc, reflète que la facture de ses importations repose plus sur les produits énergétiques qui a atteint 90686,2 millions de dirhams en 2011. Ces produits émanent essentiellement d'autres pays n'ayant pas conclu d'accord de libre-échange avec le Maroc (précisément l'Arabie Saoudite, l'Irak, la Russie, la Colombie, l'Iran, et la Guinée Equatoriale), ainsi que l'importation du blé.

Notons à cet égard que les importations du Maroc comprennent les marchandises importées d'habitude, et les marchandises importées dans le cadre du régime de l'admission temporaire pour perfectionnement actif sans paiement. 
Graphique 5 : Evolution des principaux produits à l'importation 2007-2011

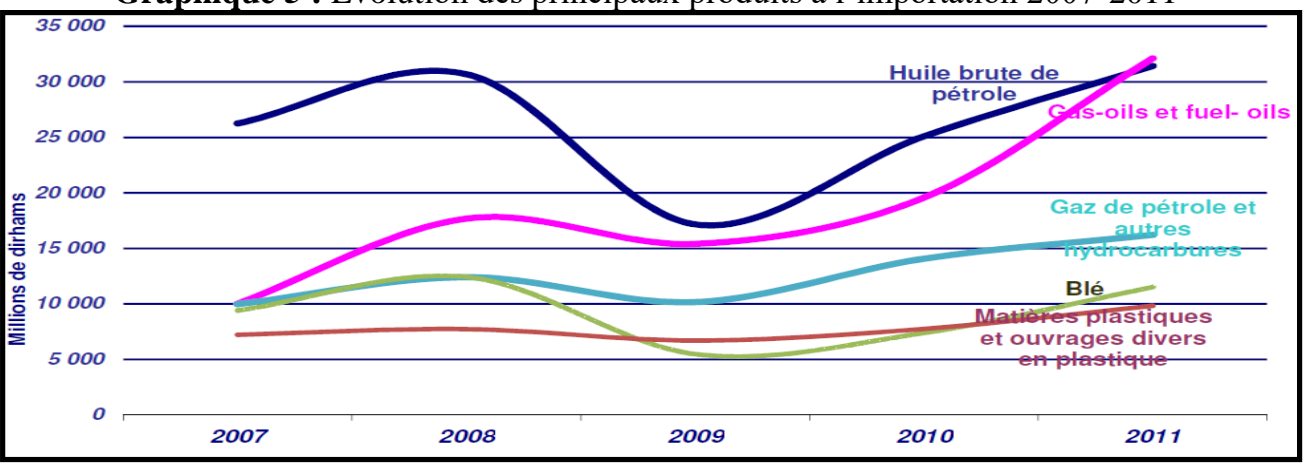

Source : Office des changes, 2013

De même, dans notre modèle de gravité, nous avons introduit la variable « taux de change » pour deux (2) raisons. La première est que le lien entre les exportations et le taux de change est bien connu en économie, et étayé par des preuves empiriques abondantes. Sharma (2003), par exemple, a montré que $10 \%$ d'une appréciation de la roupie (monnaie officielle de l'Inde) réduit la demande des exportations indiennes de 3,4\%. La deuxième raison est que le taux de change se présente comme variable explicative importante dans un certain nombre de documents de gravité, tels que Harris et Mátyás $(1998)^{23}$.

\section{III.5. Impact négligeable de la variable taux de change}

L'effet de la variation du taux de change est différent en ce qui concerne l'orientation des exportations et des importations du Maroc. Ainsi, nous constatons que cette variable n'influence pas les flux des exportations puisqu'elle n'est pas significative, alors qu'elle se trouve significative pour l'équation des importations.

A la différence de ses importations, les exportations du Maroc surtout vers les pays européens ne sont pas affectées par les variations du taux de change. En effet, les exportations marocaines vers ces pays se composent essentiellement des produits de première nécessité (produits agricoles, de la pêche, matières premières...), ainsi que les produits de confections, équipements de transport... . L'importance de ces produits, notamment les produits de première nécessité pousse l'UE, ainsi que les pays arabes de l'espace euro-méditerranéen à les importer en l'absence d'une variation importante du taux de change qui pourrait rendre les produits marocains beaucoup moins compétitifs.

${ }^{23}$ XUAN NGUYEN B. (2010), The determinants of Vietnamese export flows : static and dynamic panel gravity approaches, In International Journal of Economics and Finance, Vol. 2 No. 4, November 2010, P 3. 
En fait, rappelons que la valeur du taux de change au Maroc est déterminée en grande partie par rapport à l'Euro. Or, la crise de la dette dans la zone Euro a contribué fortement à une récession des flux des exportations du Maroc vers les pays européens et même vers les pays arabes méditerranéens avec lesquels le Maroc avait conclu des accords de libre-échange. Cette récession est relative surtout aux changements des prix des matières premières telles que le pétrole et les phosphates.

Notons que cette variable «taux de change » est d'une importance cruciale pour les importations marocaines. Cela semble évident puisque l'appréciation des monnaies de ses partenaires commerciaux rend les produits de ces pays plus chers et moins concurrentiels. Cette situation conduit à la diminution des importations marocaines à partir de ces pays.

\section{Implications et perspectives du processus d'intégration du Maroc dans l'espace euro-méditerranéen}

Les accords conclus par le Maroc dans l'espace euro-méditerranéen représentent une importante phase dans l'accomplissement du processus d'intégration dans cet espace. Il s'agit également d'un facteur d'appui à la crédibilisation du partenariat euro-méditerranéen dans son ensemble. Nous pouvons considérer ces accords comme une opportunité qui facilite l'expansion des flux des échanges et d'investissement du Maroc dans l'espace euro-méditerranéen.

Cependant, quelque soit l'importance de ces accords pour l'économie nationale, les écarts de développement entre le Maroc et ses partenaires européens, y compris la Turquie ont creusé le déficit commercial du pays avec l'UE et avec la Turquie.

En fait, il nous semble que le processus d'intégration du Maroc avec les pays du Sud de la méditerranée est une réplique à l'apparition de nouveaux concurrents du Maroc à l'échelle international, précisément sur le marché de l'UE.

Néanmoins, face à ces contraintes, le Maroc est appelé aujourd'hui à faire face à plusieurs défis afin de renforcer son intégration dans l'espace euroméditerranéen, en faisant de cette zone un groupement favorable pour le développement du potentiel des échanges nationaux.

\section{IV.1. Retombées économiques des accords de libre-échange euro- méditerranéen conclus par le Maroc}

D'après notre étude, nous avons constaté que l'UE est le premier partenaire commercial du Maroc, et que toute ouverture commerciale passe par l'Europe. Notre étude a montré la faiblesse d'une coopération Nord-Sud adoptée par le Maroc dans le cadre de ses relations euro-méditerranéennes. Ce qui démontre que l'inégalité de développement est le principal obstacle à cette 
forme d'intégration adoptée par le Maroc avec les pays du Nord de cet espace, à savoir « la zone de libre-échange ».

L'étude empirique que nous avons menée, nous a permis de conclure que l'accord de libre-échange entre le Maroc et l'UE n'est pas mutuellement bénéfique. Si cet accord représente des avantages pour l'UE, nous avons constaté qu'il n'est pas bénéfique pour le Maroc.

Cette ouverture du Maroc, et son intégration dans l'espace euroméditerranéen, ne peuvent pas se limiter à la seule UE malgré l'adoption d'un statut avancé et de différents accords à caractère commercial, notamment l'accord agricole adopté par le parlement européen après une longue négociation, l'accord sur la libéralisation des services en cours de négociation, ainsi qu'un possible accord de libre-échange approfondi et global. Mais le Maroc est appelé plus que jamais à diversifier ses partenaires commerciaux afin de réduire sa forte dépendance vis-à-vis du marché de l'UE. En fait, le processus d'intégration dans l'espace euro-méditerranéen, dont l'impact déstabilisateur, complexe et difficile parait inévitable dans un premier temps, peut être bénéfique à terme, et reste une importante issue pour que le Maroc participe activement à l'exigence de la mondialisation.

Rappelons par ailleurs que le Maroc dispose de certains atouts qu'il doit exploiter dans ses relations euro-méditerranéennes (premier producteur et exportateur de phosphates au monde par exemple), et le processus d'intégration entamé par le pays n'aurait d'autres effets que d'accroître son intensité commerciale avec ses différents partenaires régionaux. D'une part, le Maroc est un recul stratégique pour l'Europe. D'autre part, le Maroc bénéficie d'une place de choix dans certains groupements régionaux, qui le prédisposent à jouer un rôle important en matière de lutte contre certains fléaux, tel est le cas de l'immigration clandestine.

Cependant, nous avons constaté d'après notre étude que le processus d'intégration régionale adoptée par le Maroc avec les pays du Nord et ceux du Sud de la méditerranée, lui permet de bénéficier du démantèlement tarifaire sur certains produits (droits moyens préférentiels aux importations). Mais, ces accords ne favorisent pas la croissance des exportations nationales, puisque le pays se trouve concurrencé sur son principal marché, à savoir l'UE par la Chine et les Etats-Unis, qui demeurent les premiers fournisseurs des pays de l'espace euro-méditerranéen, notamment l'UE.

Notons à cet égard que la théorie économique moderne invalide empiriquement l'hypothèse de «partenaires naturels d'échange», qui considère que les accords de libre-échange sont plus appropriés entre des pays qui ont déjà un volume important d'échange commerciaux bilatéraux. L'entrée en vigueur d'un accord de libre-échange entre le Maroc et ses partenaires méditerranéens peut encourager d'autres producteurs étrangers à envisager des exportations vers les marchés ciblés par le Maroc. Ce qui finalement contribue 
à accroitre l'expansion de leurs marchés au détriment des produits marocains. Cette situation peut être expliquée par la forte concurrence mondiale imposée par la mondialisation et l'ouverture des marchés, plus précisément après la fin de l'accord multifibre en 2005 puisque le Maroc a un avantage comparatif dans ce secteur.

Toutefois, ces accords de libre-échange n'ont fait que stimuler les importations du Maroc en creusant ainsi le déficit de sa balance commerciale, d'où l'apparition d'un échange inégal entre le Maroc et ses partenaires méditerranéens, ainsi que des difficultés de résister à la forte concurrence internationale. Ce constat nous permet de dire que ces accords de libreéchange ont créé un dualisme et des inégalités d'échange entre le Maroc et ses voisins, précisément ceux du Sud avec lesquels le Maroc a opté pour des accords de libre-échange, à savoir la Tunisie, la Turquie, la Jordanie, et l'Egypte.

D'ailleurs, depuis plusieurs années, l'UE a intensifié sa présence dans la rive Sud de la méditerranée à travers la mise en place d'une Zone de libreéchange dans laquelle le Maroc s'est engagé. Or, si cette zone devait avantager les entreprises européennes qui profitent de l'ouverture du marché marocain et de l'abaissement des obstacles aux échanges avec ses partenaires commerciaux, les entreprises nationales sont appelées, à leur tour, à relever plusieurs défis, et doivent saisir les opportunités de ce processus d'ouverture ${ }^{24}$.

Cependant, malgré les efforts déployés par le Maroc pour mettre à niveau son tissu économique afin de se préparer pour l'ouverture, et faire avancer ainsi le processus d'intégration dans l'espace euro-méditerranéen, la forme d'intégration adoptée par le pays demeure toujours fragile, marquée surtout par une relation gagnant-perdant. En fait, si la concrétisation de ces efforts a avancé sur certains chapitres, elle choppe sur d'autres.

De ce fait, le Maroc est appelé aujourd'hui à redynamiser les capacités productives des entreprises nationales, en offrant un meilleur rapport qualité/prix, devenue aujourd'hui une exigence pour saisir les opportunités qu'il peut se procurer par le processus d'intégration qu'il a entamé dans l'espace euro-méditerranéen particulièrement, et dans le marché mondial généralement.

Rappelons que, les flux des importations du Maroc avec ses partenaires méditerranéens sont supérieurs à ses exportations, et que son processus d'intégration dans l'espace euro-méditerranéen a contribué à l'augmentation de ses importations. Or, les importations du Maroc comprennent même les admissions temporaires sans paiement des droits et taxes, ce qui rend la facture des importations plus importante que ses exportations. Ce constat nous permet

${ }^{24}$ Driss KHROUZ ; Partenariat euro-méditerranéen et ouverture de l'économie marocaine ; Séminaire «Pour une approche alternative de l'analyse du développement » Université Mohamed V ; 1999 ; P 109. 
de noter que le Maroc est un débouché pour les pays développés, et qu'il opte souvent pour des activités de sous-traitance, d'où l'importance de nouer de nouvelles relations commerciales avec des pays ayant son même niveau de développement.

Avec les pays du Sud euro-méditerranéen, nous pensons que le Maroc est appelé a renforcer une intégration fondée sur la complémentarité plutôt que la forte concurrence qui marque leurs relations commerciales, qui ne fait par conséquent que détériorer les chances de faire de l'espace euro-méditerranéen l'un des blocs régionaux les plus dynamiques.

Nous avons aussi constaté, que le Maroc ne favorise pas dans ses relations l'attractivité gravitationnelle «distance» dans ses échanges commerciaux. Donc, il peut nouer des relations commerciales en négligeant la contrainte de distance et de situation géographique (comme c'est le cas avec la Turquie et la Jordanie). De ce fait, la distance n'est pas un facteur déterminant de l'évaluation des partenaires éventuels de ces accords de libreéchange, puisque aujourd'hui le bouleversement de la mondialisation a permis de développer d'importants acteurs logistiques avec des offres de service diversifiées.

Au-delà de la contrainte de distance, nous avons observé une forte dépendance du Maroc vis-à-vis du marché européen, plus précisément avec ses anciens colonisateurs (la France et l'Espagne).

En fait, même après l'élargissement de l'UE, le Maroc a pu établir de nouvelles relations avec les pays nouvellement intégrés dans l'Union (Bulgarie, Roumanie...). Ce qui risque de détériorer la part de marché des produits nationaux, voir même disparaître avec la domination du marché local par les produits européens suite à la forte dépendance du Maroc de l'UE. Par ailleurs, le potentiel d'intégration du Maroc avec ses partenaires arabes se trouve aujourd'hui limité, suite à sa forte dépendance vis-à-vis de l'UE.

En effet, les échanges du Maroc avec les pays du Sud de la méditerranée avec lesquels il avait conclu des accords de libre-échange restent faibles à comparer avec les pays européens, même si le niveau de développement national est différent de celui de l'Union. Ceci valide les fondements de la théorie de l'industrie naissante dans notre cas, puisque cette dernière génère des bienfaits aux économies des pays de l'UE.

Cette théorie défend l'idée selon laquelle une industrie naissante peut, dans certaines circonstances, être un catalyseur du développement économique d'un pays, en ce sens qu'elle est source d'idées, de progrès et d'investissement dans les nouvelles techniques ${ }^{25}$. Elle confirme les résultats obtenus de notre modèle de gravité, et reflète la forte dépendance de

${ }^{25}$ BOUCHER M. (1973), List et la théorie de l'industrie naissante, In L'Actualité économique vol. $49 \mathrm{n}^{\circ} 2$ (pp 259-268). 
l'économie nationale vis-à-vis des économies de l'UE, en négligeant les avantages que le Maroc peut se procurer d'un renforcement de son processus d'intégration à travers les accords de libre-échange avec les pays du Sud et de l'Est de la méditerranée.

Dans ce contexte, le Maroc est appelé à entreprendre certaines actions sur le plan interne et externe pour bénéficier du processus d'intégration qu'il a entamé dans l'espace euro-méditerranéen. D'une part, les réformes de structure sur le plan économique qu'il avait adopté doivent être poursuivies pour rendre l'économie nationale plus compétitive et plus attractive, ainsi que le développement du capital humain sur le plan social qui devient une exigence aujourd'hui, étant donné que le capital humain est une source de développement de toute nation. D'autre part, le Maroc doit orienter son action vers la fragmentation de la dépendance et la réunion de certaines conditions pour exploiter les atouts de l'indépendance ${ }^{26}$.

De ce fait, il convient de se demander si l'espace euro-méditerranéen est un espace avantageux pour l'économie Marocaine à long terme. Si cet espace pourrait être un bloc régional à long terme qui peut concurrencer les différents blocs régionaux ayant connu une forte croissance ces dernières années, tel est le cas de l'ALENA, l'ASEAN, et qui ont contribué au développement des économies de leurs pays membres en leur offrant des opportunités importantes à saisir.

Malgré les efforts engagés par les pays de certains blocs, il se trouve que les écarts de développement entre les pays contribuent défavorablement au renforcement d'une forte intégration régionale dans une zone (le cas de l'ALENA formé par le Canada, les Etats Unis, et le Mexique). Donc, le processus d'intégration régionale est fondé sur l'équilibre de développement entre les pays partenaires, chose qui ne se manifeste pas dans le cas du Maroc en ce qui concerne son processus d'intégration dans l'espace euroméditerranéen. Ce processus reste surtout fondé sur les principes d'une coopération Nord-Sud déséquilibrée. Même avec l'existence d'une coopération Sud-Sud, il se trouve que cette relation est surtout basée sur une forte concurrence plutôt qu'une complémentarité.

Par conséquent, nous pouvons dire, sur les bases de nos constats et résultats, au long de cette partie que les accords de libre-échange adoptés par le Maroc avec le Nord et le Sud de la méditerranée n'ont pas été bénéfiques pour le pays, tant qu'ils sont basés sur une approche gagnant-perdant.

Avec les pays de l'UE, l'absence d'une convergence entre l'économie marocaine et les économies des pays de l'UE bloque l'avancement de l'accord de libre-échange avec les pays du Nord de la méditerranée. Néanmoins, avec

${ }^{26}$ ZOUITNI H. (1999), L'ancrage du Maroc à l'Europe : risques et garde-fous, Actes de colloque organisé par l'Université Caddi Ayad, Marrakech le 26 et 27 Novembre 1999. 
les pays du Sud méditerranéens, malgré l'existence d'une convergence avec les économies de ces pays, la forte concurrence qui marque les relations commerciales avec ces derniers, notamment sur le marché de l'UE, prouve la forte dépendance du Maroc vis-à-vis de l'UE d'une part, comme elle ne permet pas de faire avancer le processus d'intégration entamé avec ces pays d'autre part.

Ces constats nous permettent de remettre en question l'intérêt de cette forme d'intégration adopté par le Maroc avec les pays de l'espace euroméditerranéen, à savoir « le libre-échange ».

\section{IV.2. Ajustements pour avancer le processus d'intégration du Maroc dans l'espace euro-méditerranéen}

Face aux difficultés de réussir la forme d'intégration adoptée par le Maroc dans l'espace euro-méditerranéen que nous avons essayé de traiter, le Maroc est appelé aujourd'hui a relever plusieurs défis.

En fait, si la pierre angulaire du processus d'intégration dans cet espace est basée sur le partenariat euro-méditerranéen, ce dernier nécessite une redynamisation pour faciliter le processus d'intégration des pays en développement comme le Maroc.

Certes, le Maroc a diversifié ses partenaires dans cet espace en concluant des accords avec le Nord et avec le Sud. Cependant, le pays s'est concentré sur l'UE comme principal partenaire de cet espace.

Par ailleurs, plusieurs actions doivent être menées pour renforcer son accord avec l'UE pour que le processus d'intégration soit équilibré et avantageux pour les deux (2) partenaires.

Dans le cadre de ses relations avec l'UE, le secteur agricole a toujours été le principal secteur négocié entre les deux (2) partenaires. Cependant, il faut noter que ce secteur nécessite des débats plus profonds pour que les termes de l'échange soient mutuellement bénéfiques entre les deux (2) partenaires. Ceci nécessite un important éclaircissement dans l'accord, surtout que le déficit commercial avec l'UE se creuse de plus en plus.

Notons tout de même, que les aides financières octroyées au Maroc restent insuffisantes, et marquées par la lourdeur d'exécution des projets. Ceci nécessite un redressement du règlement des aides financières d'une part, et prévoir une planification rentable de différents projets financés d'autre part, en optant pour une démarche stratégique qui donne plus d'importance aux secteurs prioritaires pour le pays.

Donc, une révision en volume et en valeur de l'aide octroyée par le partenaire européen en faveur du Maroc est nécessaire, notamment après l'octroi d'un statut avancé au pays, et puisque les prémisses de l'accord de libre-échange complet et approfondi commence à voir le jour avec les négociations qui ont réuni les deux parties au début de l'année 2014. 
Nous pensons de ce fait, que le Maroc est appelé aujourd'hui plus que jamais, à réduire sa forte dépendance vis-à-vis de l'UE, plus précisément avec ses anciens colonisateurs, à savoir l'Espagne et la France, étant donné que les lacunes du processus d'intégration à travers l'accord de libre-échange a montré ses limites, notamment après la crise financière internationale en 2008, suivie de la récession dans la zone Euro dans le début de l'année 2010 ainsi que le printemps arabe. Ce sont tous des facteurs qui ont creusé le déficit commercial du Maroc avec les pays de l'UE et ceux du sud de la méditerranée. Ce qui nous démontre la défaillance de cette forte dépendance du Maroc visà-vis de l'UE, qualifiée comme intégration gagnant-perdant, et la défaillance des accords conclus avec le Sud de la méditerranée fondés essentiellement sur la forte concurrence entre les partenaires.

Comme il est indispensable de reconstruire les termes de coopération avec les pays arabes méditerranéens, et rassembler les secteurs de spécialisation de chaque pays autour d'un harmonieux projet régional auquel le Maroc peut s'intégrer. L'objectif principal est de créer un esprit de complémentarité entre les pays en développement tout en mobilisant leurs ressources internes au lieu d'orienter les stratégies des pays vers la forte concurrence qui demeure défaillante pour le pays.

Pour répondre à cette exigence, il nous semble qu'il est nécessaire aujourd'hui d'adapter l'appareil productif national à la demande mondiale, en favorisant les secteurs dans lesquels le Maroc est doté d'un avantage comparatif. L'objectif principal est de répondre aux nouvelles exigences de l'ouverture, et tendre vers une forme d'intégration plus poussée.

De ce fait, il est indispensable pour le Maroc de dépasser tout conflit politique, et redynamiser la coopération avec les pays Sud méditerranéens, en accordant une priorité aux objectifs commerciaux, et tendre vers une coopération Sud-Sud plus rentable.

Nous pensons dans ce cadre que le marché africain peut être un marché plus rentable pour le Maroc, vue l'importance des ressources que ses pays possèdent, qui nous semblent être substituables à certains produits importés par le pays.

Concernant ses exportations vers les pays Sud méditerranéens avec lesquels il a conclu des accords de libre-échange, nous pensons que ces accords ne peuvent être bénéfiques que si et seulement si la coopération du Maroc avec ses pays repose sur une complémentarité, en valorisant les secteurs dans lesquels le pays est doté d'un avantage comparatif.

Il nous semble que l'adoption de cette forme d'intégration avec les pays de l'espace euro-méditerranéen, à savoir le libre-échange, qui a permis l'élimination des barrières douanières avec les pays du Sud, contribuera favorablement à l'émergence des marchés avec des dimensions importantes. Ces marchés représenteront un vaste espace pour développer leurs échanges 
et former une intégration basée sur les principes d'une complémentarité de leurs marchés. Donc, le renforcement d'une intégration Sud-Sud reste essentiel dans le développement de toute la région méditerranéenne.

\section{Conclusion}

L'objectif principal de cette étude était de déterminer le potentiel des échanges du Maroc dans le cadre de l'adoption des accords de libre-échange avec les pays de l'espace euro-méditerranéen. Nous avons essayé de dégager si le processus d'intégration adopté par le Maroc avec les pays de cet espace est bénéfique pour le pays, en utilisant des données de panels en deux (2) techniques différentes, à savoir, un modèle statique et un modèle dynamique. Cette modélisation nous a permis d'évaluer l'impact des accords de libreéchange conclus par le Maroc dans cet espace.

Le modèle que nous avons construit dans cette étude a montré que, certainement, le Maroc a entamé une ouverture qui s'est manifestée par le processus d'intégration régionale autour de la méditerranée à travers la conclusion des accords de libre-échange. Toutefois, ce processus d'intégration s'est accentué par une croissance de ses flux d'importations au détriment de ses exportations. D'où la détérioration et le déficit de sa balance commerciale.

A partir des deux (2) équations de gravité proposées dans notre étude, il ressort de l'analyse effectuée que le Maroc constitue un important débouché pour les pays méditerranéens, plus précisément pour l'UE qui élargit davantage les champs d'application de son accord de libre-échange avec le Maroc. Quant à ses accords avec les pays Sud, ils sont plus marqués par leur caractère concurrentiel plutôt que complémentaire en réduisant ainsi la chance de valorisation des secteurs pour lesquels le Maroc est doté d'un avantage comparatif. Ceci nous semble être évident puisque le Maroc ne peut pas répondre aux besoins du marché européen, étant donné que ses capacités de production demeurent restreintes, alors que l'UE est considérée comme un large marché, qui rassemble 28 pays et plus de 500 millions d'habitants.

Cette analyse nous amène à une conclusion importante. Les accords de libre-échange signés par le Maroc avec les différents pays méditerranéens, permettent certainement une diminution graduelle des droits de douane et améliorent les conditions d'accès des produits nationaux sur les marchés étrangers, en renforçant ainsi le processus d'intégration dans l'espace euroméditerranéen. Or, ces accords de libre-échange ont creusé le déficit commercial du Maroc. Ceci s'explique principalement par la forte dynamique des importations suite à la conclusion de ces accords, et dont la croissance a dépassé celle des exportations.

Nous constatons que le Maroc, dans le cadre de ses relations euroméditerranéennes, repose plus sur un régionalisme qualifié « ouvert » avec la simple création de zone de libre-échange avec l'UE et avec les autres pays du 
Sud et de l'Est de la méditerranée sans développer la forme d'intégration qui le relie avec ces pays.

Globalement, nous avons conclu que les accords adoptés par le Maroc avec les pays du Nord de l'espace euro-méditerranéen ne sont pas bénéfiques pour le pays. D'une part, l'absence d'une convergence entre l'économie marocaine et les économies de l'UE, ainsi que les écarts de développement qui existent entre le pays et ses partenaires européens ne permettent pas de faire avancer le processus d'intégration d'une part. D'autre part, même avec l'existence d'un niveau de développement similaire, la coopération existante entre le Maroc et les pays arabes méditerranéens est fondée sur une concurrence et non pas sur une complémentarité. D'où l'échec du processus d'intégration adopté même avec ces pays. Il est à préciser, à cet effet, que cette concurrence s'explique par les similarités qui marquent l'offre de ces pays en termes de produits à exporter.

Si les résultats de cette étude montrent que les accords de libre-échange jouent un rôle important dans la dynamique des échanges commerciaux du Maroc avec ses partenaires euro-méditerranéens, il importe de souligner que la simple ratification de ces accords ne peut à elle seule stimuler les exportations de manière à rééquilibrer la balance commerciale du pays. Ce dernier doit plutôt veiller à l'adoption de stratégies internes de promotion des secteurs économiques à fort potentiel d'exportation.

\section{References:}

1. BLANC P. (2010), La Méditerranée sans l'Europe, Editions L'Harmattan, Paris.

2. BALASSA B. (1987), Economic integration, In The New Palgrave: A dictionary of economies, Editions Stockton Press, New York.

3. BOURBONNAIS R. (2009), Econométrie 7ème Editions, Dunod, Paris.

4. CATIN M., REGNAULT H. (2006), Le sud de la méditerranée face aux défis du libre-échange, Editions L'Harmattan, Paris.

5. DE CHARRETTE H. (2006), Pour un nouveau partenariat euroméditerranéen, Editions L'Harmattan, Paris.

6. NEWTON I., BIARNAIS M.-F (1985), De la gravitation ou les fondements de la mécanique classique, Editions Les belles Lettres, Paris.

7. SEVESTRE P. (2002), Econométrie des données de panels, Editions Dunod, Paris.

8. BOUCHER M. (1973), List et la théorie de l'industrie naissante, In L'Actualité économique, vol. 49, nº 2, pp. 259-268. 
9. LEBLOND P., ANDREA STRACHINESCU-OLTEANU M. (2009), Le libre-échange avec l'Europe : quel est l'intérêt pour le canada, In Canadian Foreign Policy Vol $15 \mathrm{~N}^{\circ} 1$, pp. 60-76.

10. MARTIN I (2010), Economic integration in the mediterranean : Beyond the 2010 free trade area, In Mediterranean Yearbook, Fundacion CIDOB/IEMed, pp. 73-78.

11. XUAN NGUYEN B. (2010), The determinants of Vietnamese export flows: static and dynamic panel gravity approaches, In International Journal of Economics and Finance Vol. 2, No. 4 November 2010, pp. 122-129.

12. BUN, M. J. G., \& KLAASSEN F. J. G. M., (2002), The importance of dynamics in panel gravity models of trade, UvA Econometrics Discussion Papers, No 2002/18, Department of Quantitative Economics, University of Amsterdam.

13. KHROUZ D. (1999), Partenariat euro-méditerranéen et ouverture de l'économie marocaine, Séminaire «Pour une approche alternative de l'analyse du développement » Université Mohamed V.

14. ZOUITNI H. (1999), L'ancrage du Maroc à l'Europe : risques et garde-fous, Actes du colloque organisé par l'Université Caddi Ayad, Marrakech le 26 et 27 Novembre 1999.

15. NGOWATTANA S (2005), Effets gravitationnels de l'AFTA sur l'ASEAN : analyse de panel, Cahiers de recherche EURIsCo $\mathrm{N}^{\circ} 2005-$ 10, Université Paris Dauphine. 